NBER WORKING PAPER SERIES

\title{
OPTIMAL INATTENTION TO THE STOCK MARKET WITH INFORMATION COSTS AND TRANSACTIONS COSTS
}

\author{
Andrew B. Abel \\ Janice C. Eberly \\ Stavros Panageas \\ Working Paper 15010 \\ http://www.nber.org/papers/w15010
}

\author{
NATIONAL BUREAU OF ECONOMIC RESEARCH \\ 1050 Massachusetts Avenue \\ Cambridge, MA 02138
}

May 2009

We thank Hal Cole, George Constantinides, Ravi Jagannathan, Ricardo Reis, Harald Uhlig, three anonymous referees and seminar participants at Duke University, New York University, Princeton University, University of British Columbia, University of Chicago, the NBER Summer Institute, Penn Macro Lunch Group, and the "Beyond Liquidity" Conference at the University of Chicago for helpful comments and discussion. The views expressed herein are those of the author(s) and do not necessarily reflect the views of the National Bureau of Economic Research.

NBER working papers are circulated for discussion and comment purposes. They have not been peerreviewed or been subject to the review by the NBER Board of Directors that accompanies official NBER publications.

(C) 2009 by Andrew B. Abel, Janice C. Eberly, and Stavros Panageas. All rights reserved. Short sections of text, not to exceed two paragraphs, may be quoted without explicit permission provided that full credit, including ( $)$ notice, is given to the source. 
Optimal Inattention to the Stock Market with Information Costs and Transactions Costs Andrew B. Abel, Janice C. Eberly, and Stavros Panageas

NBER Working Paper No. 15010

May 2009

JEL No. E21,G11

\begin{abstract}
Recurrent intervals of inattention to the stock market are optimal if consumers incur a utility cost to observe asset values. When consumers observe the value of their wealth, they decide whether to transfer funds between a transactions account from which consumption must be financed and an investment portfolio of equity and riskless bonds. Transfers of funds are subject to a transactions cost that reduces wealth and consists of two components: one is proportional to the amount of assets transferred, and the other is a fixed resource cost. Because it is costly to transfer funds, the consumer may choose not to transfer any funds on a particular observation date. In general, the optimal adjustment rule---including the size and direction of transfers, and the time of the next observation---is state-dependent. Surprisingly, unless the fixed resource cost of transferring funds is large, the consumer's optimal behavior eventually evolves to a situation with a purely time-dependent rule with a constant interval of time between observations. This interval of time can be substantial even for tiny observation costs. When this situation is attained, the standard consumption Euler equation holds between observation dates if the consumer is sufficiently risk averse.
\end{abstract}

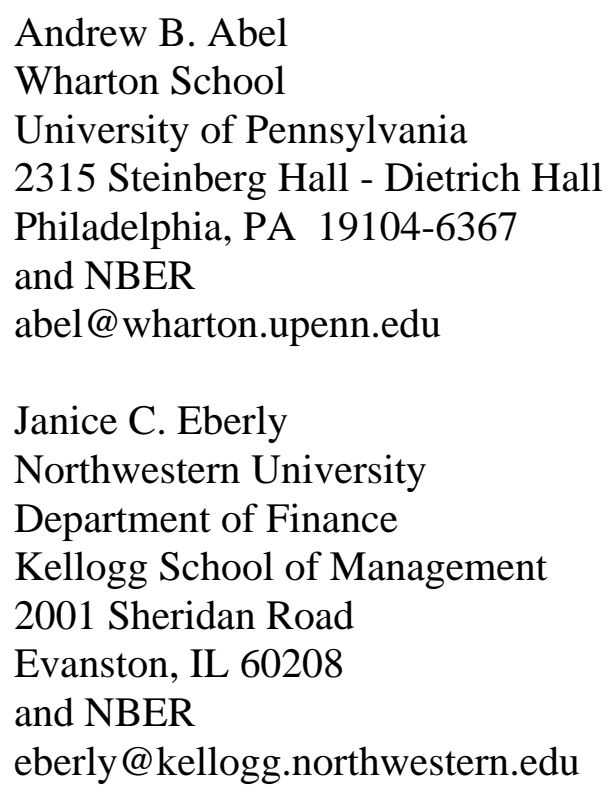

Stavros Panageas

University of Chicago - Booth School of Busines 5807 South Woodlawn Avenue

Chicago, IL, 60637

and NBER

stavros.panageas@chicagobooth.edu 
A pervasive finding in studies of microeconomic choice is that adjustment to economic news tends to be sluggish and infrequent. Investors rebalance their portfolios and revisit their spending behavior at discrete and potentially infrequent points of time. Between these times, inaction is the rule. If individuals take several months or even years to adjust their portfolios and their spending plans, the standard predictions of the consumption smoothing and portfolio choice theories might fail, and the standard intertemporal Euler equation relating asset returns and consumption growth may not hold. Similar sorts of inaction also characterize the financing, investment, and pricing behavior of firms. These observations have led economists to formulate models that are consistent with infrequent adjustment. ${ }^{1}$

Formal models of infrequent adjustment are often described as either time-dependent or state-dependent. In time-dependent models, adjustment is triggered simply by calendar time. In state-dependent models, adjustment takes place only when a particular state variable reaches some trigger value, so the timing of adjustments depends on factors other than, or in addition to, calendar time alone. A classic example of state-dependent adjustment is the $(\mathrm{S}, \mathrm{s})$ model. The distinction between time-dependent and state-dependent models can have crucial implications for important economic questions. For instance, monetary policy has substantial real effects that persist for several quarters if firms change their prices according to a time-dependent rule. However, if firms adjust their prices according to a state-dependent rule, then monetary policy may have little or no effect on the real economy. (See e.g. Caplin and Spulber (1987) and Golosov and Lucas (2007).)

In this paper we develop and analyze an optimizing model that can generate both time-dependent adjustment and state-dependent adjustment. The economic context is an infinite-horizon continuous-time model of consumption and portfolio choice that builds on the framework of Merton (1971). We augment Merton's model by requiring consumption to be purchased with the liquid asset and by introducing two sorts of costs - a utility cost of observing the value of the consumer's wealth; and a resource cost of transferring assets between a transactions account consisting of liquid assets and an investment portfolio consisting of risky equity and riskless bonds. We motivate the utility cost of observing the value of wealth as a reduction in leisure associated with obtaining and analyzing information. ${ }^{2}$ We model the resource cost of transferring assets as the sum of two components: (1) a component that is proportional to the amount of assets transferred; and (2) a component that is a homogeneous linear function of the balances in the transactions account and in the investment portfolio. Since the second component is independent of the amount of assets transferred, we refer to it as a fixed resource cost of transferring assets. 
Because it is costly to observe the value of wealth, the consumer chooses to observe this value only at discretely-spaced points in time. At these observation times, the consumer chooses when next to observe the value of wealth, executes any transfers between the investment portfolio and the transactions account, and chooses the path of consumption until the next observation date. During intervals of time between consecutive observations, optimal behavior is characterized by inattention to the value of the consumer's wealth, in particular, to the value of equities.

In general, the timing of asset transfers is state-dependent. The relevant state of the consumer's balance sheet at time $t$ is the ratio of the balance in the transactions account to the contemporaneous value of the investment portfolio. We denote this ratio as $x_{t}$. The consumer may or may not transfer assets between the investment portfolio and the transactions account on an observation date $t_{j}$ depending on the value of $x_{t_{j}}$. Because the timing of asset transfers depends on the value of $x_{t_{j}}$, these transfers are state-dependent. A surprising result of our analysis, however, is that, provided the fixed resource cost of transferring assets is not large, eventually an optimally inattentive consumer's asset transfers are purely time-dependent. Indeed, when asset holdings get to this stage, the optimal time between successive asset transfers is constant.

When the consumer has relatively large holdings in the transactions account on an observation date, he will transfer some of these assets to the investment portfolio. Alternatively, when the consumer's holdings in the transactions account are low on an observation date, he will sell some assets from the investment portfolio to replenish the transactions account in order to finance consumption until the next observation date. However, when the transactions account has an intermediate balance, measured by an intermediate value of $x_{t_{j}}$ on an observation date, the consumer will not find it worthwhile to pay the costs associated with transferring assets between the investment portfolio and the transactions account. We show that eventually optimal behavior by a consumer facing observation costs leads to a low value of $x_{t_{j}}$ on an observation date. Once a low value of $x_{t_{j}}$ is realized on an observation date, the consumer transfers only enough assets to the transactions account to finance consumption until the next observation date, provided that the fixed resource cost of transferring assets is not too large. This behavior is optimal because it is costly to transfer assets, and the liquid asset in the transactions account earns a lower rate of return than the riskless bond in the investment portfolio. In this case, the consumer plans to hold a zero balance in the transactions account on the next observation date, so that $x_{t_{j}}$ will equal zero on the next observation date. Thus, on the next observation date, $x_{t_{j}}$ will again have a low value and 
the situation repeats itself.

This paper is related to two strands of literature. The first strand is the large literature on transactions costs. In Baumol (1952) and Tobin (1956), which are the forerunners of the cash-in-advance model used in macroeconomics, consumers can hold two riskless assets that pay different rates of return: money, which pays zero interest, and a riskless bond that pays a positive rate of interest. As in our paper, consumers are willing to hold money, despite the fact that its rate of return is dominated by the rate of return on riskless bonds, because goods have to be purchased with money. That is, money offers liquidity services.

A more recent literature on portfolio transactions costs, including Constantinides (1986) and Davis and Norman (1990), models the cost of transferring assets between stocks and bonds in the investment portfolio as proportional to the size of the transfers. Here we also include proportional transactions costs, but these costs apply only to transfers between the liquid asset in the transactions account on the one hand and the investment portfolio of stocks and bonds on the other. We do not model the costs of reallocating stocks and bonds within the investment portfolio. For a retired consumer who finances consumption by withdrawing assets from a tax-deferred retirement account, the cost of withdrawing assets from the investment portfolio includes taxes paid at the time of withdrawal. For most consumers in this situation, the marginal tax rate, which is part of the cost of transferring assets from the investment portfolio to the transactions account, is likely to be far greater than any costs associated with reallocating stocks and bonds within the investment portfolio.

A second strand of the literature analyzes the infrequent adjustment of choice variables that arises because it is costly to observe and process information. This strand of literature itself has proceeded in two directions. One direction, which includes Sims (2003) and Moscarini (2004), uses the information-theoretic concept of entropy in a linear-quadratic framework to model rational inattention as the outcome of the limited ability of people to process information. In those papers, the decisionmaker generally receives noisy information about important state variables and can choose the timing and information content of these signals. ${ }^{3}$

The other direction pursued in the literature on infrequent adjustment does not build on directly on entropy, but instead, specifies costs of obtaining and processing information. The two closest antecedents to our current paper ${ }^{4}$ are Duffie and Sun (1990) and Abel, Eberly, and Panageas (2007). ${ }^{5}$ These papers, as well as the current paper require consumption to be purchased with a liquid asset, such as cash. In addition, because these papers include an observation cost, the consumer will not continuously observe the value of the stock market. 
In our current paper, we show that when $x_{t_{j}}$ is low, the consumer will plan to arrive at the next observation date with a zero balance in the transactions account, and that the length of time between subsequent observations is constant if the fixed cost of transferring assets is sufficiently small. Duffie and Sun derive inattention intervals of constant length, but they implicitly confine attention to low values of $x_{t_{j}}$ by explicitly assuming that $x_{t_{j}}=0$ on the first observation date. Here we show that optimal behavior is potentially different for intermediate and high values of $x_{t_{j}}$ - situations not considered by Duffie and Sun. Moreover, we show conditions under which eventually $x_{t_{j}}$ will indeed become low on an observation date and optimal behavior converges to time-dependence. In this sense, Duffie and Sun confine attention to the long run for the case with low fixed costs of transferring assets, while we consider the transition path to the long run and long-run behavior, as well as the conditions necessary for this absorption to occur. Importantly, consideration of behavior outside of the long-run situation allows the model to incorporate state-dependent adjustment as well as purely time-dependent adjustment. In addition, we also offer a quantitative assessment of the length of the interval of time between consecutive observations in the long run. Finally, relative to our own earlier paper, the current paper explicitly allows separate consideration of observation costs and transactions costs and models the observation cost as a utility cost rather than a resource cost. ${ }^{6,7}$

Section 1 sets up the consumer's decision problem. Section 2 characterizes the optimal trigger and return values for the state variable $x_{t}$. In addition, this section contains a detailed discussion of a typical indifference curve of the value function to illustrate various aspects of optimal adjustment behavior. The dynamic evolution of $x_{t}$ is analyzed in Section 3, which also characterizes the long-run situation that is eventually attained if the fixed component of transactions costs is sufficiently small. In addition, Section 3 presents a numerical illustration of the constant length of time between consecutive observations in the long run, followed by a discussion of the Euler equation. Section 4 concludes. The Appendix contains proofs of various results.

\section{Consumer's Decision Problem}

Consider an infinitely-lived consumer who does not earn any labor income but has wealth that consists of risky equity, riskless bonds, and a riskless liquid asset. Consumption must be purchased with the liquid asset, which the consumer holds in a transactions account. Risky equity and riskless bonds are held in an investment portfolio and cannot be used directly 
to purchase consumption. The consumer is not permitted to take either a leveraged or a negative position in equity.

\subsection{Asset Returns}

Equity is a non-dividend-paying stock with a price $P_{t}$ that evolves according to a geometric Brownian motion

$$
\frac{d P_{t}}{P_{t}}=\mu d t+\sigma d z,
$$

where $\mu>0$ is the mean rate of return and $\sigma$ is the instantaneous standard deviation. The riskless bond in the investment portfolio has a constant instantaneous rate of return $r_{f}$ that is positive and less than the mean rate of return on equity, so $0<r_{f}<\mu$. The total value of the investment portfolio, consisting of equity and riskless bonds, is $S_{t}$ at time $t$.

At time $t$, the consumer holds $X_{t}$ in the liquid asset, which pays a riskless instantaneous rate of return $r_{L}$, where $r_{L}<r_{f}$. The rate of return on the liquid asset, $r_{L}$, is lower than the rate of return on the riskless bond in the investment portfolio, $r_{f}$, because the liquid asset provides transactions services not provided by the bond in the investment portfolio.

Suppose the consumer observes the value of the investment portfolio at time $t_{j}$ and next observes its value at time $t_{j+1}=t_{j}+\tau_{j}$. Immediately upon observing the values of $S_{t_{j}}$ and $X_{t_{j}}{ }^{8}$ the consumer may transfer assets between the investment portfolio and the liquid asset in the transactions account (at a cost described below) so that at time $t_{j}^{+}$the value of the investment portfolio is $S_{t_{j}^{+}}$. The consumer chooses to hold a fraction $\phi_{j}$ of $S_{t_{j}^{+}}$in risky equity and a fraction $1-\phi_{j}$ in riskless bonds and does not rebalance the investment portfolio before the next observation. ${ }^{9}$ Since the consumer cannot take a negative position in equity and cannot take a leveraged position in equity, we have $0 \leq \phi_{j} \leq 1$. When the consumer next observes the value of the investment portfolio, at time $t_{j+1}=t_{j}+\tau_{j}$, its value is

$$
S_{t_{j+1}}=R\left(\tau_{j}\right) S_{t_{j}^{+}}
$$

where

$$
R\left(\tau_{j}\right) \equiv \phi_{j} \frac{P_{t_{j+1}}}{P_{t_{j}}}+\left(1-\phi_{j}\right) e^{r_{f} \tau_{j}}
$$

is the gross rate of return on the investment portfolio over the $j-$ th interval of time, which extends from $t_{j}^{+}$to $t_{j+1}=t_{j}+\tau_{j}$. 


\subsection{Costs of Transferring Assets}

The consumer can transfer assets between the investment portfolio and the transactions account by incurring a resource cost that is proportional to the size of the transfer and a "fixed" resource cost that is independent of the size of the asset transfer. Specifically, if the consumer sells $-y^{s} \geq 0$ dollars of assets from the investment portfolio, there is a proportional transfer cost of $-\psi_{s} y^{s}$ dollars, where $0 \leq \psi_{s}<1$, so that a sale of $-y^{s}$ dollars from the investment portfolio is accompanied by an increase in $X$ of $-\left(1-\psi_{s}\right) y^{s}$ dollars. For transfers in the other direction, in which the consumer uses the transactions account to buy additional assets in the investment portfolio, a purchase of $y^{b} \geq 0$ dollars in the investment portfolio is accompanied by a decrease in $X$ of $\left(1+\psi_{b}\right) y^{b}$ dollars, where $\psi_{b} \geq 0$. We assume that $\psi_{s}+\psi_{b}>0$ so that at least one of the proportional transfer cost parameters is positive. Perhaps the most obvious interpretation of the proportional transactions costs, $\psi_{s}$ and $\psi_{b}$, is that they represent brokerage fees. Another interpretation presents itself if we consider the investment portfolio to be a tax-deferred account such as a 401k account. In this case, the consumer must pay a tax on withdrawals from the investment portfolio, and $\psi_{s}$ would include the consumer's income tax rate, which would be substantially higher than a brokerage fee. ${ }^{10}$

The fixed resource cost is independent of the size of the asset transfer but is a linear function of the amounts of assets held in the transactions account and in the investment portfolio. Specifically, the fixed resource cost is $\theta_{X} X+\theta_{S} S$, where $0 \leq \theta_{X}<<1$ and $0 \leq \theta_{S}<1-\psi_{s}{ }^{11}$ We assume that $\theta_{X} X$ is paid from the transactions account and $\theta_{S} S$ is paid from the investment portfolio. Of course, if $\theta_{X}=\theta_{S}$, the fixed resource cost is simply proportional to total wealth $X+S .{ }^{12}$

Define

$$
x \equiv \frac{X}{S}
$$

as the ratio of the transactions account to the investment portfolio. It will be convenient to calculate the change in $S$ that accompanies a given change in $x$ when the consumer transfers assets between the investment portfolio and the transactions account. Specifically, suppose that the consumer arrives at observation date $t_{j}$ with $\left(X_{t_{j}}, S_{t_{j}}\right)$, so that $x_{t_{j}}=X_{t_{j}} / S_{t_{j}}$. If the consumer buys $y^{b} \geq 0$ dollars of assets in the investment portfolio or sells $-y^{s} \geq 0$ dollars

of assets from the investment portfolio, the new vector of holdings is $\left(X_{t_{j}^{+}}, S_{t_{j}^{+}}\right)$where

$$
X_{t_{j}^{+}}=\left[1-\left(\mathbf{1}_{\left\{y^{b}>0\right\}}+\mathbf{1}_{\left\{y^{s}<0\right\}}\right) \theta_{X}\right] X_{t_{j}}-\left(1+\psi_{b}\right) y^{b}-\left(1-\psi_{s}\right) y^{s}
$$


and

$$
S_{t_{j}^{+}}=\left[1-\left(\mathbf{1}_{\left\{y^{b}>0\right\}}+\mathbf{1}_{\left\{y^{s}<0\right\}}\right) \theta_{S}\right] S_{t_{j}}+y^{b}+y^{s},
$$

where $\mathbf{1}_{\left\{y^{b}>0\right\}}$ is an indicator function that equals 1 if $y^{b}>0$ and equals 0 otherwise, and $\mathbf{1}_{\left\{y^{s}<0\right\}}$ is an indicator function that equals 1 if $y^{s}<0$ and equals 0 otherwise. Use equations (5) and (6) to obtain ${ }^{13}$

$$
\frac{S_{t_{j}^{+}}}{S_{t_{j}}}=\frac{\left[1-\left(\mathbf{1}_{\left\{y^{b}>0\right\}}+\mathbf{1}_{\left\{y^{s}<0\right\}}\right) \theta_{X}\right] x_{t_{j}}+\left[1-\left(\mathbf{1}_{\left\{y^{b}>0\right\}}+\mathbf{1}_{\left\{y^{s}<0\right\}}\right) \theta_{S}\right]\left[\left(1+\psi_{b}\right) \mathbf{1}_{\left\{y^{b}>0\right\}}+\left(1-\psi_{s}\right) \mathbf{1}_{\left\{y^{s}<0\right\}}\right]}{x_{t_{j}^{+}}+\left(1+\psi_{b}\right) \mathbf{1}_{\left\{y^{b}>0\right\}}+\left(1-\psi_{s}\right) \mathbf{1}_{\left\{y^{s}<0\right\}}} .
$$

\subsection{The Utility Function}

\subsubsection{Consumption between Observation Dates}

Suppose that the consumer observes the value of the investment portfolio only at discretelyspaced points in time $t_{0}, t_{1}, t_{2}, \ldots$. At observation date $t_{j}$, after observing the value of the investment portfolio, lifetime utility is

$$
E_{t_{j}}\left\{\int_{t_{j}}^{\infty} \frac{1}{1-\alpha} c_{t}^{1-\alpha} e^{-\rho\left(t-t_{j}\right)} d t-\sum_{i=j+1}^{\infty} A_{i} e^{-\rho\left(t_{i}-t_{j}\right)}\right\}
$$

where $c_{t}$ is consumption at time $t, A_{i}$ is the utility cost of observing the investment portfolio at time $t_{i}, 0<\alpha \neq 1$, and the rate of time preference, $\rho>0$, is large enough so that

$$
e^{-\rho \tau_{j}} E_{t_{j}}\left\{\left[R\left(\tau_{j}\right)\right]^{1-\alpha}\right\}<1 \text {. }
$$

We will specify the observation cost $A_{i}$ more precisely in subsection 1.3.2. For now, treat $A_{i}$ as exogenous.

Once the consumer observes the value of assets at date $t_{j}$, he will not observe any new information until the next observation date, $t_{j+1}$. Therefore, at time $t_{j}$, the consumer can plan the entire path of consumption from time $t_{j}^{+}$to time $t_{j+1}$. Let $C\left(t_{j}, \tau_{j}\right)$ be the present

value, discounted at rate $r_{L}$, of the flow of consumption over the interval of time from $t_{j}^{+}$ until the next observation date, $t_{j+1} \equiv t_{j}+\tau_{j}$. Specifically,

$$
C\left(t_{j}, \tau_{j}\right)=\int_{t_{j}^{+}}^{t_{j+1}} c_{s} e^{-r_{L}\left(s-t_{j}\right)} d s,
$$

where the path of consumption $c_{s}, t_{j}^{+} \leq s \leq t_{j+1}$, is chosen to maximize the discounted value of utility over the interval from $t_{j}^{+}$to $t_{j+1}$. Let

$$
U\left(C\left(t_{j}, \tau_{j}\right)\right)=\max _{\left\{c_{s}\right\}_{s=t_{j}^{+}}^{a_{j+1}}} \int_{t_{j}^{+}}^{t_{j+1}} \frac{1}{1-\alpha} c_{s}^{1-\alpha} e^{-\rho\left(s-t_{j}\right)} d s,
$$


subject to a given value of $C\left(t_{j}, \tau_{j}\right)$ in equation (10). Since the consumer does not observe any new information between time $t_{j}^{+}$and time $t_{j+1}$, the maximization in equation (11) is a standard intertemporal optimization under certainty. It is straightforward to show that ${ }^{14}$

$$
\begin{gathered}
U\left(C\left(t_{j}, \tau_{j}\right)\right)=\frac{1}{1-\alpha}\left[h\left(\tau_{j}\right)\right]^{\alpha}\left[C\left(t_{j}, \tau_{j}\right)\right]^{1-\alpha}, \\
C\left(t_{j}, \tau_{j}\right)=h\left(\tau_{j}\right) c_{t_{j}^{+}},
\end{gathered}
$$

and

$$
U^{\prime}\left(C\left(t_{j}, \tau_{j}\right)\right)=c_{t_{j}^{+}}^{-\alpha},
$$

where

$$
h\left(\tau_{j}\right) \equiv \int_{0}^{\tau_{j}} e^{-\omega s} d s=\frac{1-e^{-\omega \tau_{j}}}{\omega}
$$

and we assume that

$$
\omega \equiv \frac{\rho-(1-\alpha) r_{L}}{\alpha}>0 .
$$

Since all of the consumption during the interval of time from $t_{j}^{+}$to $t_{j+1}$ is financed from the liquid asset in the transactions account, which earns an instantaneous riskless rate of return $r_{L}$, we have

$$
X_{t_{j+1}}=e^{r_{L} \tau_{j}}\left(X_{t_{j}^{+}}-C\left(t_{j}, \tau_{j}\right)\right) .
$$

\subsubsection{Cost of Observing the Value of the Investment Portfolio}

Now we specify the observation cost $A_{i}$ in more detail. We motivate the specification of $A_{i}$ by thinking of the observation cost as foregone leisure and building on a utility formulation widely used in the real business cycle and growth literatures. Specifically, in those literatures, instantaneous utility at time $t$ is specified as $u\left(c_{t}, l_{t}\right)=\frac{1}{1-\alpha} c_{t}^{1-\alpha} v\left(l_{t}\right)$, where $c_{t}$ is the flow of consumption at time $t$ and $l_{t}$ is the flow of leisure at time $t .{ }^{15}$ If we were to adopt this formulation directly, we would specify $v\left(l_{t}\right)=1$ at times that the consumer does not observe the value of the investment portfolio and $v\left(l_{t}\right)=1-(1-\alpha) \theta_{1}, \theta_{1}>0$, at observation times $t_{j}$, reducing instantaneous utility by a multiplicative factor at observation times. Specifically, the utility cost of observing the investment portfolio at time $t_{j+1}$ would be $A_{j+1}=\theta_{1} c_{t_{j+1}}^{1-\alpha}$. However, if the observation cost were specified in this way, an optimizing consumer would plan a discontinuous jump in consumption at time $t_{j+1}$ in order to reduce the observation cost. We regard this planned discontinuity in the path of consumption in the absence of new information as an unattractive feature. Therefore, we adopt an alternative specification of the observation cost that avoids this problem but yields the same value of the observation cost. Specifically, suppose that immediately after observing the value of the investment 
portfolio at time $t_{j}$, the consumer chooses the path of consumption from $t_{j}^{+}$to $t_{j+1}$ ignoring any effect of consumption on the observation cost $A_{j+1}$. In this case, the standard Euler equation for a deterministic intertemporal consumption problem implies (see equation $*$ in footnote 14) that $c_{t_{j+1}}=e^{-\frac{\rho-r_{L}}{\alpha} \tau_{j}} c_{t_{j}^{+}}$so that the utility cost can be written as

$$
A_{j+1}=\theta_{1} e^{-(1-\alpha) \frac{\rho-r_{L}}{\alpha} \tau_{j}} c_{t_{j}^{+}}^{1-\alpha}
$$

Equations (12) and (13) imply

$$
c_{t_{j}^{+}}^{1-\alpha}=(1-\alpha) \frac{U\left(C\left(t_{j}, \tau_{j}\right)\right)}{h\left(\tau_{j}\right)} .
$$

Substitute equation (19) into equation (18), multiply both sides of the resulting equation by $e^{-\rho \tau_{j}}$, and use the definition of $h(\tau)$ in equation to (15) to obtain the present value of $A_{j+1}$ discounted back to time $t_{j}$

$$
e^{-\rho \tau_{j}} A_{j+1}=\theta_{1}(1-\alpha) \frac{U\left(C\left(t_{j}, \tau_{j}\right)\right)}{e^{\omega \tau_{j}} h\left(\tau_{j}\right)} .
$$

We adopt equation (20) as the fundamental expression for the discounted value of the observation cost $A_{j+1}$. Because $A_{j+1}$ is proportional to the integral of utility from consumption over the interval $\left(t_{j}, t_{j+1}\right]$, it does not distort the path of consumption over this interval. In particular, an optimizing consumer will plan to have a continuous path of consumption over this entire interval.

Use equation (20) and the expression for lifetime utility in (8) to obtain the value function at observation date $t_{j}$. The value function, immediately after observing the value of the investment portfolio at date $t_{j}$, can be represented recursively by

$$
\begin{aligned}
V\left(X_{t_{j}}, S_{t_{j}}\right)= & \max _{C\left(t_{j}, \tau_{j}\right), y^{b}, y^{s}, \phi_{j}, \tau_{j}}\left(1-\frac{\theta_{1}(1-\alpha)}{e^{\omega \tau_{j}} h\left(\tau_{j}\right)}\right) U\left(C\left(t_{j}, \tau_{j}\right)\right) \\
& +\beta^{\tau_{j}} E_{t_{j}}\left\{V\left(e^{r_{L} \tau_{j}}\left(X_{t_{j}^{+}}-C\left(t_{j}, \tau_{j}\right)\right), R\left(\tau_{j}\right) S_{t_{j}^{+}}\right)\right\} .
\end{aligned}
$$

where $\beta^{\tau_{j}} \equiv e^{-\rho \tau_{j}}$ and the maximization in equation (21) is subject to equations (5) and (6) and the inequality constraints $C\left(t_{j}, \tau_{j}\right) \leq X_{t_{j}^{+}}, 0 \leq \phi_{j} \leq 1, y^{b} \geq 0$, and $y^{s} \leq 0$.

The value function in equation (21) is homogeneous of degree $1-\alpha$ in $X_{t_{j}}$ and $S_{t_{j}}$, and consequently the optimal length of time between consecutive observations, $\tau_{j}$, is a function of $x_{t_{j}}$. Because the value function is homogeneous of degree $1-\alpha$ in $X_{t_{j}}$ and $S_{t_{j}}$, it can be written as

$$
V\left(X_{t_{j}}, S_{t_{j}}\right)=\frac{1}{1-\alpha} S_{t_{j}}^{1-\alpha} v\left(x_{t_{j}}\right)
$$


Define the marginal rate of substitution at observation date $t_{j}$ between $X_{t_{j}}$ and $S_{t_{j}}$ as

$$
m\left(x_{t_{j}}\right) \equiv \frac{V_{S}\left(X_{t_{j}}, S_{t_{j}}\right)}{V_{X}\left(X_{t_{j}}, S_{t_{j}}\right)} .
$$

The expression for $V\left(X_{t_{j}}, S_{t_{j}}\right)$ in equation (22) can be used to rewrite the marginal rate of substitution in equation (23) as

$$
m\left(x_{t_{j}}\right)=\frac{(1-\alpha) v\left(x_{t_{j}}\right)}{v^{\prime}\left(x_{t_{j}}\right)}-x_{t_{j}} .
$$

\section{Trigger and Return Values of $x$}

The value of $x_{t_{j}} \equiv \frac{X_{t_{j}}}{S_{t_{j}}}$ on an observation date $t_{j}$ determines whether, and in which direction, the consumer transfers any assets between the investment portfolio and the transactions account. There are two trigger values of $x, \omega_{1}$ and $\omega_{2}$, that determine whether the consumer transfers assets, and there are two return values of $x, \pi_{1}$ and $\pi_{2}$, that characterize the optimal value of $x_{t_{j}^{+}}$immediately after a transfer.

To define and characterize the trigger values, $\omega_{1}$ and $\omega_{2}$, we first define the restricted value function $\widetilde{V}\left(X_{t_{j}}, S_{t_{j}}\right)$ at observation date $t_{j}$ as the maximized expected value of utility over the infinite future, subject to the restriction that the consumer does not transfer any assets between the transactions account and the investment portfolio at time $t_{j}$ (but optimally transfers assets between the transactions account and the investment portfolio at all future observation dates). Formally,

$$
\begin{aligned}
\widetilde{V}\left(X_{t_{j}}, S_{t_{j}}\right)= & \max _{C\left(t_{j}, \tau_{j}\right), \phi_{j}, \tau_{j}}\left(1-\frac{\theta_{1}(1-\alpha)}{e^{\omega \tau_{j}} h\left(\tau_{j}\right)}\right) U\left(C\left(t_{j}, \tau_{j}\right)\right) \\
& +\beta^{\tau_{j}} E_{t_{j}}\left\{V\left(e^{r_{L} \tau_{j}}\left(X_{t_{j}}-C\left(t_{j}, \tau_{j}\right)\right), R\left(\tau_{j}\right) S_{t_{j}}\right)\right\},
\end{aligned}
$$

subject to $C\left(t_{j}, \tau_{j}\right) \leq X_{t_{j}^{+}}$and $0 \leq \phi_{j} \leq 1$. For the remainder of this section, we will suppress the time subscripts, with the understanding that the results apply at any observation date.

Like the value function, the restricted value function is homogeneous of degree $1-\alpha$ and can be written as

$$
\widetilde{V}(X, S)=\frac{1}{1-\alpha} S^{1-\alpha} \widetilde{v}(x) .
$$

On any observation date, $\widetilde{V}(X, S) \leq V(X, S)$, with equality only if the optimal values of $y^{b}$ and $y^{s}$ are both zero. 
Define

$$
\omega_{1} \equiv \inf x>0: \widetilde{v}(x)=v(x)
$$

and

$$
\omega_{2} \equiv \sup x>0: \widetilde{v}(x)=v(x) .
$$

The proposition below shows that $\omega_{1}$ and $\omega_{2}$ are trigger values for $x$ in the sense that if $x$ is less than $\omega_{1}$ on an observation date, the consumer will transfer assets to the transactions account, and if $x$ exceeds $\omega_{2}$ on an observation date, the consumer will transfer assets to the investment portfolio. To ensure that $\omega_{2}$ is finite, we assume that $\theta_{X}$ is not too large. Specifically, we assume

$$
\theta_{X}<\overline{\theta_{X}} \equiv(1+\nu)^{-1}\left(1+\nu^{-1}\right)^{-\nu}\left[1+(1-\alpha) \omega \theta_{1}\right]^{-\frac{\nu}{1-\alpha}}\left[\left(1-\theta_{S}\right) \frac{1-\psi_{s}}{1+\psi_{b}}\right]^{\nu}<1,
$$

where $\nu \equiv \frac{\omega}{r_{f}-r_{L}}>0$. We also define

$$
\pi_{1} \equiv \sup \left\{\begin{array}{ll}
x \geq 0: \forall z \in\left(0, \frac{x S}{1-\psi_{s}}\right], & (1) V(x S, S) \geq V\left(x S-\left(1-\psi_{s}\right) z, S+z\right) \\
\text { and }(2) V(x S, S)>\widetilde{V}\left(x S-\left(1-\psi_{s}\right) z, S+z\right)
\end{array}\right\}
$$

and

$$
\pi_{2} \equiv \inf \left\{\begin{array}{r}
x \geq 0: \forall z \in(0, S], \quad(1) V(x S, S) \geq V\left(x S+\left(1+\psi_{b}\right) z, S-z\right) \\
\text { and }(2) V(x S, S)>\widetilde{V}\left(x S+\left(1+\psi_{b}\right) z, S-z\right)
\end{array}\right\} .
$$

The proposition below shows that $\pi_{1}$ and $\pi_{2}$ are the return values for $x$. Specifically, if $x \leq \omega_{1}$, the consumer will transfer enough assets from the investment portfolio to the transactions account to increase $x$ to $\pi_{1}$. Alternatively, if $x \geq \omega_{2}$, the consumer will use the transactions account to buy enough assets in the investment portfolio to decrease $x$ to $\pi_{2}$.

Proposition 1 1. $0<\omega_{1} \leq \pi_{1} \leq \pi_{2} \leq \omega_{2}<\infty$

2. If $x_{t_{j}}<\omega_{1}$, then

(a) $y^{s}<0$

(b) $x_{t_{j}^{+}}=\pi_{1}$

(c) $m\left(x_{t_{j}}\right)=\left(1-\psi_{s}\right) \frac{1-\theta_{S}}{1-\theta_{X}}$

(d) $v\left(x_{t_{j}}\right)=\left[\frac{\left(1-\theta_{X}\right) x_{t_{j}}+\left(1-\theta_{S}\right)\left(1-\psi_{s}\right)}{\left(1-\theta_{X}\right) \omega_{1}+\left(1-\theta_{S}\right)\left(1-\psi_{s}\right)}\right]^{1-\alpha} v\left(\omega_{1}\right)$ 
3. $v\left(\omega_{1}\right)=\left[\frac{\left(1-\theta_{X}\right) \omega_{1}+\left(1-\theta_{S}\right)\left(1-\psi_{s}\right)}{\pi_{1}+1-\psi_{s}}\right]^{1-\alpha} v\left(\pi_{1}\right)$

4. If $x_{t_{j}}>\omega_{2}$, then

(a) $y^{b}>0$

(b) $x_{t_{j}^{+}}=\pi_{2}$

(c) $m\left(x_{t_{j}}\right)=\left(1+\psi_{b}\right) \frac{1-\theta_{S}}{1-\theta_{X}}$

(d) $v\left(x_{t_{j}}\right)=\left[\frac{\left(1-\theta_{X}\right) x_{t_{j}}+\left(1-\theta_{S}\right)\left(1+\psi_{b}\right)}{\left(1-\theta_{X}\right) \omega_{2}+\left(1-\theta_{S}\right)\left(1+\psi_{b}\right)}\right]^{1-\alpha} v\left(\omega_{2}\right)$

5. $v\left(\omega_{2}\right)=\left[\frac{\left(1-\theta_{X}\right) \omega_{2}+\left(1-\theta_{S}\right)\left(1+\psi_{b}\right)}{\pi_{2}+1+\psi_{b}}\right]^{1-\alpha} v\left(\pi_{2}\right)$

Proposition 1 is proved in the appendix. Here we use the indifference curves in Figure 1 to illustrate this proposition and the definitions of the trigger and return points. For simplicity, Figure 1 is drawn for the case in which $\theta_{X}=\theta_{S}$. The indifference curve of the value function $V(X, S)$ passes through points $A, B, C, D, E$, and $F$, and the indifference curve of the restricted value function $\widetilde{V}(X, S)$ passes through points $K, B, C, D, E$, and $J$. In Regions II, III, and IV, the two indifference curves are identical so that the marginal rate of substitution between $X$ and $S$ is the same for both the value function and the restricted value function. In addition, $V(X, S)=\widetilde{V}(X, S)$ in these regions so that the maximized value of expected lifetime utility is obtained by the restricted value function, which assumes that the consumer does not transfer any assets between the investment portfolio and the transactions account on the current observation date. Therefore, Regions II, III, and IV represent the "inaction region" in which the consumer optimally chooses not to transfer assets between the investment portfolio and the transactions account.

The consumer will transfer assets if $V(X, S)>\widetilde{V}(X, S)$, which is the case in Regions I and V. For instance, in Region I, the indifference curve of the restricted value function passes through point $B$ and lies above the indifference curve of the value function that also passes through point $B$, thereby implying that $V(X, S)>\widetilde{V}(X, S)$ in this region. ${ }^{16}$ In order to attain the maximized value of expected lifetime utility, the consumer must transfer assets between the investment portfolio and the transactions account. As shown in statement 2a of Proposition 1, $y^{s}<0$ so the consumer sells assets from the investment portfolio to increase the amount of liquid assets in the transactions account. Similarly, according to statement $4 \mathrm{a}$, if the consumer is in Region $\mathrm{V}$ on an observation date, the optimal policy is to use some of the liquid assets in the transactions account to purchase additional assets in the investment portfolio. 


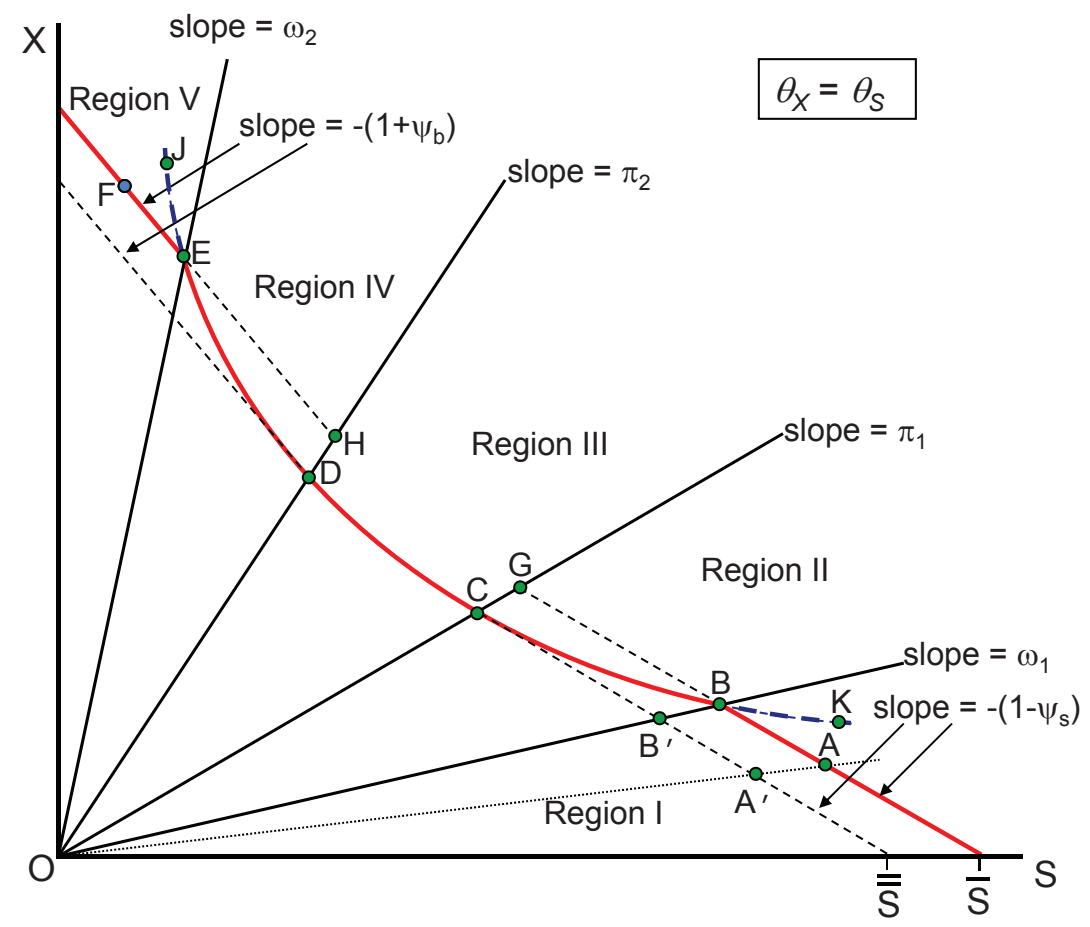

Figure 1: Indifference Curve of the Value Function When $\theta_{X}=\theta_{S}$.

Now consider the return value $\pi_{1}$. We proceed in two steps. First, assume that the consumer has already paid the fixed transfer cost $\theta_{2}(X+S)$, where $\theta_{2}$ is the common value of $\theta_{X}=\theta_{S}$, and that the consumer is choosing the size of the asset transfer from the investment portfolio to the transactions account. In the second step, we consider the impact of the fixed transfer cost, $\theta_{2}(X+S)$, on the optimal transfer.

Suppose that, after paying the fixed cost $\theta_{2}(X+S)$, the consumer is located somewhere to the right of point $C$ along the dashed line through point $C$ with slope $-\left(1-\psi_{s}\right)$. For instance, suppose that the consumer is at point $A^{\prime}$. Having already paid the fixed cost, the consumer can move instantaneously to any point up and to the left of point $A^{\prime}$ along the dashed line with slope $-\left(1-\psi_{s}\right)$ by reducing $S$ by $-y^{s}>0$ dollars and increasing $X$ by $\left(1-\psi_{s}\right)\left(-y^{s}\right)$ dollars. The consumer will sell assets from the investment portfolio, until $(X, S)$ reaches point $C$, where the dashed line with slope $-\left(1-\psi_{s}\right)$ is tangent to the indifference curve, which is essentially a smooth-pasting condition. At point $C$, the ratio of $X$ to $S$, i.e., $x$, is equal to $\pi_{1}$, as indicated by the line through points $O, C$, and $G$, which has slope equal to $\pi_{1}$.

Now let's consider the impact of the fixed cost $\theta_{2}(X+S)$ on the optimal transfer of assets. If $\theta_{2}>0$, the consumer cannot move from point $A^{\prime}$ to point $C$. To see the impact 
of $\theta_{2}>0$, consider the line through points $G, B$, and $A$, which is parallel to the line through points $C, B^{\prime}$, and $A^{\prime}$, and hence has slope $-\left(1-\psi_{s}\right)$. Point $G$ lies on the half-line through the origin with slope $\pi_{1}$ and is located so that the length of $\overline{O C}$ is $1-\theta_{2}$ times the length of $\overline{O G}$. The properties of similar triangles imply that the length of $\overline{O B^{\prime}}$ is $1-\theta_{2}$ times the length of $\overline{O B}$ and that the length of $\overline{O A^{\prime}}$ is $1-\theta_{2}$ times the length of $\overline{O A}$.

Now suppose that the consumer starts at point $A$ and sells $-y^{s}>0$ dollars from the investment portfolio, thereby incurring a cost of $\theta_{2}(X+S)-\psi_{s} y^{s}$ dollars. The fixed cost of $\theta_{2}(X+S)$ dollars reduces both $X$ and $S$ by the fraction $\theta_{2}$ and can be represented by the movement from point $A$ to point $A^{\prime}$; the sale of $-y^{s}>0$ dollars from the investment portfolio can be represented by a movement from point $A^{\prime}$ upward and leftward along the dashed line through points $C, B^{\prime}$, and $A^{\prime}$. The consumer will be willing to move from $A$ to point $C$ only if doing so increases (or at least does not lower) the value of the value function. That is, the gain in value from moving to an improved allocation between $X$ and $S$, with $x=\pi_{1}$, must outweigh the fixed cost $\theta_{2}(X+S)$ represented by the movement downward and leftward from the line through points $G, B$, and $A$ to the line through points $C, B^{\prime}$, and $A^{\prime}$. For a large change in the ratio $x$, such as the change in moving from point $A$ to point $C$, the net gain in value is positive. For a small change in $x$, the change is not worthwhile. At point $B$, the gain from the improved allocation between $X$ and $S$ is exactly offset by the cost of moving from the line through points $G, B$, and $A$ to the line through points $C, B^{\prime}$, and $A^{\prime}$. Formally, this equality of gain and benefit is represented by statement 3 in Proposition 1 , which is essentially a value-matching condition.

For points along the segment $\overline{G B}$, the change in the value of $x$ is small enough that the improved allocation between $X$ and $S$ is outweighed by the fixed cost $\theta_{2}(X+S)$. Therefore, the consumer will not transfer assets from any points along this segment. The fact that the consumer will not move from points along segment $\overline{G B}$ to point $C$ is illustrated by the fact that these points lie above the indifference curve of the value function that passes through point $C$. Alternatively, for points below and to the right of point $B$ along the line through points $A$ and $B$, the improved asset allocation made possible by moving to point $C$, and the associated increase in value, are large enough to compensate for the fixed transfer cost, and the consumer will move from any of these points to $C$ (statements $2 \mathrm{a}$ and $2 \mathrm{~b}$ ). Since the consumer ends up at the same point, namely point $C$, from any point below and to the right of point $B$, all of these points have the same value. Thus, all of these points lie on the same indifference curve (statement $2 \mathrm{~d}$ ), so that indifference curve has slope equal to $-\left(1-\psi_{s}\right)$ below and to the right of point $B$, which is statement $2 \mathrm{c}$ in Proposition $1 .{ }^{17}$

We have used Figure 1 to illustrate the trigger point $\omega_{1}$ and the return point $\pi_{1}$ when the 
consumer chooses to sell assets from investment portfolio. A similar set of arguments can explain the trigger point $\omega_{2}$ and the return point $\pi_{2}$ when the consumer chooses to use some of the liquid assets in the transactions account to buy assets in the investment portfolio.

We conclude this section with the following corollary to Proposition 1.

Corollary $1 \omega_{1} \leq x_{t_{j}^{+}} \leq \omega_{2}$.

This corollary states that the value of $x_{t}$ immediately following any observation date $t_{j}$ (and following any optimal asset transfers at date $t_{j}^{+}$) is confined to the closed interval $\left[\omega_{1}, \omega_{2}\right]$. This result will be useful when we analyze the dynamic behavior of asset holdings in the next section.

\section{Dynamic Behavior}

We have shown that the direction of the optimal transfer on an observation date depends on the value of $x_{t_{j}}$. In this section, we examine the dynamic behavior of $X$ and $S$ between observation dates. Because no new information arrives between observation dates and the rate of return on the transactions account is riskless, the evolution of $X$ between observation dates is deterministic. By contrast, the value of the investment portfolio evolves stochastically over time. If the value of $X$ is positive on the subsequent observation date, then, depending on the outcome of the stochastic process for $S$, the consumer potentially could be in any of the five regions on the next observation date. However, if $\theta_{X}$ and $\theta_{S}$ are sufficiently small, the stochastic process for $x_{t_{j}}$ will eventually be absorbed at $x_{t_{j}}=0$.

Proposition 2 For sufficiently small values of $\theta_{X} \geq 0$ and $\theta_{S} \geq 0$, if $x_{t_{j}}<\omega_{1}$ on observation date $t_{j}$, then $x_{t_{k}}=0$ on all subsequent observation dates $t_{k}>t_{j}$.

The proof of Proposition 2 is in the Appendix. Here we provide an intuitive argument. First, consider the case in which $\theta_{X}=\theta_{S}=0$. If $x_{t_{j}}<\omega_{1}$ on observation date $t_{j}$, the optimal transfer is to sell assets from the investment portfolio to increase the transactions account so that $x_{t_{j}^{+}}$increases to $\pi_{1}$. Since each additional dollar that is sold from the investment portfolio incurs a transactions cost $\psi_{s}$, and since the transactions account earns a lower riskless rate of return than the riskless rate of return on bonds in the investment portfolio, the consumer would never sell more assets from investment portfolio than are needed to finance consumption until the next observation date. Thus, the consumer will arrive at the

next observation date with zero liquid assets, so that $x_{t_{j+1}}$ will be zero. Since $x_{t_{j+1}}=0<\omega_{1}$, 
the process will repeat itself ad infinitum with $x_{t_{k}}=0$ on every observation date $t_{k}>t_{j}$ and $x_{t_{k}^{+}}=\pi_{1}$ immediately after every future observation date.

If $\theta_{X}$ and $\theta_{S}$ are positive, then we need to consider the possibility that the consumer would want to arrive at the next observation date with enough liquid assets in the transactions account to avoid transferring assets from the investment portfolio and thus avoid paying the fixed transactions cost at that date. As we prove in the Appendix, if $\theta_{X}$ and $\theta_{S}$ are small enough, the consumer will still optimally choose to arrive at the next observation date with a zero balance in the transactions account, even though this action necessitates payment of the fixed transaction cost at the next observation date.

The following lemma together with Proposition 2 allows us to prove that the stochastic process for $x_{t_{j}}$ is eventually absorbed at zero, if the fixed cost of transferring assets is sufficiently small.

Lemma 1 Eventually, $x_{t_{j}}<\omega_{1}$ on an observation date.

The proof of Lemma 1 is in the Appendix. Here we provide an intuitive argument. Because the expected rate of return on equity, $\mu$, exceeds the riskless rate of return, $r_{f}$, on bonds in the investment portfolio, the optimal share of equity, $\phi_{j}$, is positive (see Lemma 4 ). Therefore, there is a chance that $R\left(\tau_{j}\right)$ will be sufficiently high that $x_{t_{j+1}}=\frac{e^{r_{L} \tau_{j}}\left(X_{t_{j}^{+}}-C\left(t_{j}, \tau_{j}\right)\right)}{R\left(\tau_{j}\right) S_{t_{j}^{+}}}$ will be less than $\omega_{1}$. Eventually, this event will occur.

Proposition 3 For sufficiently small values of $\theta_{X} \geq 0$ and $\theta_{S} \geq 0$, the stochastic process for $x_{t_{j}}$ is eventually absorbed at zero.

Proof. Lemma 1 states that eventually $x_{t_{j}}<\omega_{1}$ on an observation date. Proposition 2 implies that when this event occurs, $x_{t_{j+1}}=0$ on the next observation date and on all subsequent observation dates, provided that $\theta_{X} \geq 0$ and $\theta_{S} \geq 0$ are sufficiently small.

Corollary 2 For sufficiently small $\theta_{X} \geq 0$ and $\theta_{S} \geq 0$, in the long run: (a) $x_{t_{j}}=0$ on every observation date and (b) the consumer sells assets from the investment portfolio so that $x_{t_{j}^{+}}=\pi_{1}$ immediately after every observation date.

Proposition 3 and its corollary imply that, provided the fixed transaction cost parameters $\theta_{X}$ and $\theta_{S}$ are sufficiently small, the consumer will eventually reach a repeating pattern in which a constant amount of time elapses between consecutive observations of the stock market. The consumer will arrive at each observation date having just exhausted the liquid 


\begin{tabular}{lcccc}
\hline \hline & $\begin{array}{c}(1) \\
\text { Observation cost } \\
\text { (dollar equivalent) }\end{array}$ & $\begin{array}{c}(2) \\
\tau^{*}, \theta_{2}=0 \\
\text { (years) }\end{array}$ & $\begin{array}{c}(3) \\
\theta_{2}^{*} \times 10^{6} \\
\text { (dollar equivalent) }\end{array}$ & $\begin{array}{c}(4) \\
\tau^{*}, \theta_{2}=\theta_{2}^{*} \\
\text { (years) }\end{array}$ \\
\hline Baseline & 2.3 & 0.096 & 6.6 & 0.191 \\
$\theta_{1}=0.001$ & 23.3 & 0.308 & 63.0 & 0.590 \\
$\rho=0.02$ & 2.6 & 0.097 & 7.4 & 0.193 \\
$\alpha=3$ & 2.3 & 0.092 & 6.1 & 0.176 \\
$r_{L}=0$ & 2.3 & 0.079 & 11.4 & 0.195 \\
$r_{f}=0.03$ & 2.8 & 0.084 & 27.0 & 0.279 \\
$\mu=0.07$ & 2.6 & 0.088 & 6.1 & 0.161 \\
$\sigma=0.2$ & 2.1 & 0.096 & 6.3 & 0.198 \\
\hline \hline
\end{tabular}

TABLE 1: $\theta_{2}^{*}$ is the largest value of $\theta_{2}=\theta_{X}=\theta_{S}$ that leads to constant optimal inattention spans. Baseline Parameters: $\alpha=4, \rho=0.01, r_{L}=0.01, r_{f}=0.02, \mu=0.06, \sigma=0.16, \theta_{1}=0.0001$.

assets in the transactions account and will liquidate just enough assets from the investment portfolio to finance consumption until the next observation date. Thus, the consumer's behavior has a Baumol-Tobin flavor to it. The model in Duffie and Sun (1990) shares this property because it assumes that the consumer starts with $x_{t}=0$. Our results are also consistent with Duffie and Sun, despite some differences in the details of modeling of the observation costs and the fixed component of transfer costs.

Proposition 3 and its corollary are remarkable because they imply that even though the choices of observation dates and transactions dates follow state-dependent rules in general, they will eventually converge to pure time-dependent rules, provided that the fixed transactions costs are small. Table 1 presents the optimal time between consecutive observation dates in the long run for the case in which $\theta_{X}=\theta_{S}=\theta_{2}$ for the baseline parameter values reported in the table's caption. To present the observation cost and the fixed component of the transactions cost in terms of dollars, we assume that the consumer has $\$ 1$ million in the investment portfolio on an observation date. The observation cost in column (1) is the dollar equivalent of the reduction in utility associated with the observation cost. ${ }^{18}$ In the baseline case, the observation cost is $\$ 2.30$ per observation. Column (2) reports the optimal time between consecutive observations when $\theta_{2}=0$ so that fixed cost parameters $\theta_{X}$ and $\theta_{S}$ are both zero. The time between observations is measured in years, so in the baseline case, the optimal time between observations is slightly longer than one month. Column (3) reports $\theta_{2}^{*}$, which is the largest value of $\theta_{X}=\theta_{S}=\theta_{2}$ that is small enough to ensure that eventually the time between consecutive observations is constant. The values reported in column (3) are actually $\theta_{2}^{*} \times 10^{6}$ so that, for instance, in the baseline case, the fixed 
transactions cost is $\$ 6.60$ for a millionaire. Finally, column (4) reports the time between consecutive observations when $\theta_{2}=\theta_{2}^{*}$.

Table 1 allows us to draw two broad conclusions. First, even tiny observation costs can lead to substantial inattention intervals. Column (2) shows that even when the fixed costs of transacting are zero $\left(\theta_{X}=\theta_{S}=0\right)$, a consumer who owns one million dollars, and incurs an observation cost equivalent to about two dollars, will observe her portfolio at approximately a monthly frequency. Second, fixed transaction costs can significantly magnify the effect of observation costs to produce even larger inattention spans. The inattention spans in column (4) are about twice as large as the inattention spans in column (2). Intuitively, when fixed transaction costs are not too large compared to the observation costs, the consumer will find it optimal to synchronize transaction and observation dates, in order to avoid "wasting" observation costs without using the obtained information to undertake a transaction. Because of this synchronization, the optimal inattention interval is determined as if fixed transaction costs and observation costs are bundled together, effectively magnifying the impact of the observation cost. For instance, with an observation cost of $\$ 2.30$, the optimal time between observations can be more than two months, if $\theta_{2}=\theta_{2}^{*}$.

The calculations reported in Table 1 are invariant to the proportional transaction cost parameters $\psi_{b}$ and $\psi_{s}$. The irrelevance of $\psi_{b}$ results from the fact that in the long run the consumer does not ever transfer any assets from the transactions account to the investment portfolio and thus never incurs any cost $\psi_{b} y^{b}$. The proposition below implies that $\psi_{s}$ is also irrelevant for calculating the optimal time between observations in the long run when $\theta_{X}$ and $\theta_{S}$ are sufficiently small.

Proposition 4 Define $V\left(0, S_{t_{j}} ; \psi_{s}\right)$ as the value function, for a given value of the transactions cost parameter $\psi_{s}$, on observation date $t_{j}$ when $\left(X_{t_{j}}, S_{t_{j}}\right)=\left(0, S_{t_{j}}\right)$, and define $\pi_{1}\left(\psi_{s}\right)$ as the optimal return value of $x_{t_{j}^{+}}$for $x_{t_{j}}<\omega_{1}$. Suppose that $\theta_{X}$ and $\theta_{S}$ are sufficiently small that for any admissible value of $\psi_{s}$, if $x_{t_{j}}<\omega_{1}$ on observation date $t_{j}$, then on all subsequent observation dates $x_{t_{j+1}}=0$.

1. $V\left(0, S_{t_{j}} ; \psi_{s}\right)=\left(1-\psi_{s}\right)^{1-\alpha} V\left(0, S_{t_{j}} ; 0\right)$

2. the optimal observation dates $t_{k}=t_{j}+(k-j) \tau^{*}$, for $k \geq j$, are invariant to $\psi_{s}$

3. $\pi_{1}\left(\psi_{s}\right)=\left(1-\psi_{s}\right) \pi_{1}(0)$.

The proof of this proposition is in the Appendix. The intuitive argument underlying this proposition is that when $X_{t_{j}}=0$ on an observation date, so that all of the consumer's 
wealth is in the investment portfolio, $S_{t_{j}}$, the consumer can only pay for future consumption by selling assets from the investment portfolio and incurring the proportional transaction cost $\psi_{s}$. In effect, $\psi_{s}$ is a pure consumption tax that does not distort the intertemporal profile of consumption; it simply shifts the entire profile of consumption downward by a fraction $\psi_{s}$, thereby multiplying lifetime utility by $\left(1-\psi_{s}\right)^{1-\alpha}$, which is statement 1 of Proposition 4. The value of $\psi_{s}$ does not affect the timing of observations and transactions in the long run (statement 2 of Proposition 4), nor does it affect the amount of assets transferred out of the investment portfolio. However, because each dollar transferred out of the investment portfolio yields only $1-\psi_{s}$ dollars of liquid assets, $x_{t_{j}^{+}}=\pi_{1}\left(\psi_{s}\right)=\left(1-\psi_{s}\right) \pi_{1}(0)$, which is statement 3 of Proposition 4.

Optimal consumption and transfers of assets imply an Euler equation relating the intertemporal marginal rate of substitution and excess returns on equity between two observation dates. ${ }^{19}$ We prove the following proposition in the Appendix.

Proposition 5 If $\theta_{X}$ and $\theta_{S}$ are sufficiently small, and $\alpha>\frac{\mu-r_{f}}{\sigma^{2}}$, then in the long run we have $E_{t_{j}}\left\{c_{t_{j+1}^{+}}^{-\alpha}\left(\frac{P_{t_{j+1}}}{P_{t_{j}}}-e^{r_{f} \tau_{j}}\right)\right\}=0$.

Proposition 5 states that if the fixed transactions costs, parameterized by $\theta_{X}$ and $\theta_{S}$, are sufficiently small, then for sufficiently risk-averse consumers, the relevant Euler equation in the long run is the same as the standard Euler equation, provided that attention is confined to observation dates. ${ }^{20}$

\section{Concluding Remarks}

Rules governing infrequent adjustment are typically categorized as time-dependent or statedependent. Time-dependent rules depend only on calendar time and can optimally result from costs of gathering and processing information. State-dependent rules depend on the value of some state variable, typically reaching some trigger threshold, and can be the optimal response to a transactions cost. Our model combines costly information and costly transactions. In general, on any observation date, the consumer chooses the length of time until the next date at which to gather information and re-optimize, but that length of time may be state-dependent. Moreover, conditional on the information observed at that future date, the agent's action (or lack thereof) may also be state-dependent. Thus, the model has elements of both state- and time-dependent rules in general.

In the long run, however, the optimal behavior converges to a rule that is purely timedependent, provided that the fixed component of the transfer costs is sufficiently small. 
Once the consumer arrives at an observation date with a sufficiently small balance in the transactions account, he will optimally choose to arrive at all subsequent observation dates with zero liquid assets in the transactions account. In our model, this behavior results from the facts that (1) the consumer can save on costs by synchronizing observation and transactions dates and (2) the consumer would prefer to hold as little as possible of his wealth in the liquid asset because the return on the transactions account is dominated by the return on the investment account.

We have emphasized the tendency toward pure time-dependence in our model, but there are forces that could prevent this situation from arising, even within the model. If the fixed component of the transactions cost is large, then our proof that the consumer will choose to arrive at the next observation date with zero liquid assets no longer holds. And if the consumer arrives at an observation date with a positive amount of liquid assets, then the state variable $x_{t}$ could potentially take on any positive value, and a purely time-dependent rule would not be optimal even in the long run. Outside the model, one might consider allowing for the arrival of labor income in the transactions account or the occurrence of attention-grabbing events that occur when the consumer is not at a planned observation date. $^{21}$

Even though such modifications undermine convergence to a purely time-dependent rule, the fundamental forces driving this result still seem to create a tendency to exhaust (or keep a very low balance in) the liquid account: the transactions account is dominated in rate of return, and synchronizing observations and transactions reduces costs. Therefore, the consumer would routinely find himself in region I in the long run and would repeatedly transfer enough assets from the investment account to arrive at $x_{t_{j}}^{+}=\pi_{1}$. Since the inattention span $\tau$ is a function of $x_{t_{j}}^{+}$, the distributions of inattention intervals would have a point mass at $\tau\left(\pi_{1}\right)$. In the special case of a purely time-dependent rule, that point mass is equal to one. 


\section{A Appendix}

Proof of Proposition 1. We start by proving the following Lemma.

Lemma 2 Optimal behavior requires $y^{s} y^{b}=0$. If the optimal asset transfer increases $x$, then $y^{s}<0$. If the optimal transfer decreases $x$, then $y^{b}>0$.

Proof of Lemma 2. To prove that $y^{s} y^{b}=0$, suppose $y^{s} y^{b} \neq 0$, which implies that $y^{s}<0$ and $y^{b}>0$. Now consider reducing $y^{b}$ by $\varepsilon$ and increasing $y^{s}$ by $\varepsilon$, which will have no effect on the value of $S$ relative to the original transfer but will increase $X$ by $\left(\psi_{s}+\psi_{b}\right) \varepsilon$ relative to the original transfer by reducing the amount of proportional transactions cost incurred. Therefore, it could not have been optimal for $y^{s} y^{b} \neq 0$. Hence, $y^{s} y^{b}=0$.

The value function $V(X, S)$ is strictly increasing in $X$ and $S$, so an optimal transfer will never decrease both $X$ and $S$. Therefore, if the optimal transfer increases $x \equiv \frac{X}{S}$, then the optimal transfer cannot decrease $X$ and must decrease $S$, which implies that $y^{b}=0$ and $y^{s}>0$. Similarly, if the optimal transfer decreases $x \equiv \frac{X}{S}$, then the optimal transfer cannot decrease $S$ and must decrease $X$, which implies that $y^{s}=0$ and $y^{b}>0$.

Proof of statement 2a. Suppose that $x<\omega_{1}$. The definition of $\omega_{1}$ in equation (27) implies that $v(x) \neq \widetilde{v}(x)$. The optimal asset transfer will change the value of $x$ to some value $z$ for which $v(z)=\widetilde{v}(z)$. The definition of $\omega_{1}$ implies that such a $z$ cannot be less than $\omega_{1}$, so the optimal transfer increases $x$. Lemma 2 implies that $y^{s}<0$.

Proof of statement $\mathbf{2 b}$. Start from $\left(X_{0}, S_{0}\right)$ where $X_{0}<\omega_{1} S_{0}$. Statement 2a implies that optimal $y^{s}<0$. Let $\overline{y^{s}}$ be the optimal value of $y^{s}$. Therefore,

$$
V\left(\left(1-\theta_{X}\right) X_{0}-\left(1-\psi_{s}\right) \overline{y^{s}},\left(1-\theta_{S}\right) S_{0}+\overline{y^{s}}\right)=\widetilde{V}\left(\left(1-\theta_{X}\right) X_{0}-\left(1-\psi_{s}\right) \overline{y^{s}},\left(1-\theta_{S}\right) S_{0}+\overline{y^{s}}\right)
$$

and

$$
\begin{aligned}
& V\left(\left(1-\theta_{X}\right) X_{0}-\left(1-\psi_{s}\right) \overline{y^{s}},\left(1-\theta_{S}\right) S_{0}+\overline{y^{s}}\right) \\
\geq & V\left(\left(1-\theta_{X}\right) X_{0}-\left(1-\psi_{s}\right)\left(\overline{y^{s}}-\zeta\right),\left(1-\theta_{S}\right) S_{0}+\overline{y^{s}}-\zeta\right),
\end{aligned}
$$

for $\zeta \in\left[0,\left(1-\theta_{S}\right) S_{0}+\overline{y^{s}}\right]$.

Define $y^{s *}$ as the value of $y^{s}$ that will lead to $x_{0^{+}}=\pi_{1}$. Use equation (7) to show that

$$
y^{s *}=S_{0^{+}}-\left(1-\theta_{S}\right) S_{0}=\left[\frac{\left(1-\theta_{X}\right) x_{0}-\left(1-\theta_{S}\right) \pi_{1}}{\pi_{1}+1-\psi_{s}}\right] S_{0} .
$$

From this point onward, the proof proceeds by contradiction. Assume $\overline{y^{s}}>y^{s *}$ so that the magnitude of the transfer $\overline{y^{s}}$ is smaller than the transfer needed to increase $x_{0^{+}}$to $\pi_{1}$. Since $\overline{y^{s}}$ is 
optimal, equations (A.1) and (A.2) imply that

$$
\begin{aligned}
\widetilde{V}\left(\left(1-\theta_{X}\right) X_{0}-\left(1-\psi_{s}\right) \overline{y^{s}},\left(1-\theta_{S}\right) S_{0}+\overline{y^{s}}\right) & =V\left(\left(1-\theta_{X}\right) X_{0}-\left(1-\psi_{s}\right) \overline{y^{s}},\left(1-\theta_{S}\right) S_{0}+\overline{y^{s}}\right) \\
& \geq V\left(\left(1-\theta_{X}\right) X_{0}-\left(1-\psi_{s}\right) y^{s *},\left(1-\theta_{S}\right) S_{0}+y^{s *}\right)
\end{aligned}
$$

But definition of $\pi_{1}$ implies that

$\widetilde{V}\left(\left(1-\theta_{X}\right) X_{0}-\left(1-\psi_{s}\right) \overline{y^{s}},\left(1-\theta_{S}\right) S_{0}+\overline{y^{s}}\right)<V\left(\left(1-\theta_{X}\right) X_{0}-\left(1-\psi_{s}\right) y^{s *},\left(1-\theta_{S}\right) S_{0}+y^{s *}\right)$,

which contradicts equation (A.3).

Proof of statement 2c. Consider the point $\left(X_{0}, S_{0}\right)$ with $x_{0} \equiv \frac{X_{0}}{S_{0}}=\omega_{1}$ and define $D$ as the set of $(X, S)$ for which $x<\omega_{1}$ and from which the consumer can instantaneously move to $\left(X_{0}, S_{0}\right)$ by selling assets from the investment portfolio. Specifically,

$$
D \equiv\left\{\begin{array}{c}
(X, S) \text { with } X<\omega_{1} S: \\
\exists y^{s}<0 \text { for which }\left(1-\theta_{X}\right) X-\left(1-\psi_{s}\right) y^{s}=X_{0} \text { and }\left(1-\theta_{S}\right) S+y^{s}=S_{0}
\end{array}\right\} .
$$

Define $F$ as the set of $(X, S)$ for which $x \geq \omega_{1}$ and to which the consumer can instantaneously move from any point in $D$ by selling assets from the investment portfolio. Specifically,

$$
F \equiv\left\{\begin{array}{c}
(X, S) \text { with } X \geq \omega_{1} S: \\
\exists y^{s}<0 \text { for which } X=X_{0}-\left(1-\psi_{s}\right) y^{s} \text { and } S=S_{0}+y^{s} \geq 0
\end{array}\right\} .
$$

Consider two arbitrary points $\left(X_{1}, S_{1}\right)$ and $\left(X_{2}, S_{2}\right)$ in set $D$. Since $x_{1}<\omega_{1}$ and $x_{2}<\omega_{1}$, the optimal value of $y^{s}$ will be strictly negative starting from either point. Moreover, $y^{s}$ must be large enough in absolute value so that the post-transfer value of $(X, S)$ satisfies $x \equiv \frac{X}{S} \geq \omega_{1}$ because it is always optimal to sell assets from the investment portfolio from any point in set $D$. Therefore, the post-transfer value of $(X, S)$ will be an element of set $F$. Thus, regardless of whether the consumer starts from point $\left(X_{1}, S_{1}\right)$ or $\left(X_{2}, S_{2}\right)$, the consumer's choice of asset transfer can be described as choosing $\left(X^{+}, S^{+}\right) \in F$ to maximize the value function. Therefore, $V\left(X_{1}, S_{1}\right)=V\left(X_{2}, S_{2}\right)$, so all of the points in set $D$ lie on the same indifference curve of $V(X, S)$. The slope of this indifference curve is $\frac{d X}{d S}=\frac{d X}{d y^{s}} \frac{d y^{s}}{d S}=-\left(1-\psi_{s}\right) \frac{1-\theta_{S}}{1-\theta_{X}}$, which proves statement 2c.

Proof of statement 2d. We have shown that if $x<\omega_{1}$, then $m(x)=\left(1-\psi_{s}\right) \frac{1-\theta_{S}}{1-\theta_{X}}$. Using the expression for the marginal rate of substitution in equation (24) yields the differential equation

$$
\frac{(1-\alpha) v(x)}{v^{\prime}(x)}-x=\left(1-\psi_{s}\right) \frac{1-\theta_{S}}{1-\theta_{X}}, \text { for } 0 \leq x<\omega_{1},
$$

which implies

$$
v(x)=\left[\frac{\left(1-\theta_{X}\right) x+\left(1-\theta_{S}\right)\left(1-\psi_{s}\right)}{\left(1-\theta_{X}\right) \omega_{1}+\left(1-\theta_{S}\right)\left(1-\psi_{s}\right)}\right]^{1-\alpha} v\left(\omega_{1}\right), \text { for } 0 \leq x \leq \omega_{1} .
$$

Proof of statement 1. We start by proving the following Lemma. 
Lemma 3 For sufficiently small $\bar{x}>0, \frac{1}{1-\alpha} \widetilde{v}(x)<\frac{1}{1-\alpha} v(x)$ for all $x \in(0, \bar{x})$.

Proof of Lemma 3. Substitute the expression for $U\left(C\left(t_{j}, \tau_{j}\right)\right)$ from equation (12) into the restricted value function in equation (25) to obtain

$$
\begin{aligned}
\widetilde{V}\left(X_{t_{j}}, S_{t_{j}}\right)= & \max _{C\left(t_{j}, \tau_{j}\right), \phi_{j}, \tau_{j}}\left(1-\frac{\theta_{1}(1-\alpha)}{e^{\omega \tau_{j}} h\left(\tau_{j}\right)}\right) \frac{1}{1-\alpha}\left[h\left(\tau_{j}\right)\right]^{\alpha}\left[C\left(t_{j}, \tau_{j}\right)\right]^{1-\alpha} \\
& +\beta^{\tau_{j}} E_{t_{j}}\left\{V\left(e^{r_{L} \tau_{j}}\left(X_{t_{j}}-C\left(t_{j}, \tau_{j}\right)\right), R\left(\tau_{j}\right) S_{t_{j}}\right)\right\} .
\end{aligned}
$$

Use equation (13) to rewrite the first term on the right hand side of equation (A.8) as

$$
\left(1-\frac{\theta_{1}(1-\alpha)}{e^{\omega \tau_{j}} h\left(\tau_{j}\right)}\right) \frac{1}{1-\alpha}\left[h\left(\tau_{j}\right)\right]^{\alpha}\left[C\left(t_{j}, \tau_{j}\right)\right]^{1-\alpha}=\frac{1}{1-\alpha} c_{t_{j}^{+}}^{-\alpha} C\left(t_{j}, \tau_{j}\right)-\frac{\theta_{1}}{e^{\omega \tau_{j}}} c_{t_{j}^{+}}^{1-\alpha} .
$$

Substitute equation (A.9) into equation (A.8) to obtain

$\widetilde{V}\left(X_{t_{j}}, S_{t_{j}}\right)=\max _{C\left(t_{j}, \tau_{j}\right), \phi_{j}, \tau_{j}} \frac{1}{1-\alpha} c_{t_{j}^{+}}^{-\alpha} C\left(t_{j}, \tau_{j}\right)-\frac{\theta_{1}}{e^{\omega \tau_{j}}} c_{t_{j}^{+}}^{1-\alpha}+\beta^{\tau_{j}} E_{t_{j}}\left\{V\left(e^{r_{L} \tau_{j}}\left(X_{t_{j}}-C\left(t_{j}, \tau_{j}\right)\right), R\left(\tau_{j}\right) S_{t_{j}}\right)\right\}$.

Because the choice of $C\left(t_{j}, \tau_{j}\right)$ must satisfy the constraint $X_{t_{j}}-C\left(t_{j}, \tau_{j}\right) \geq 0$, the partial derivative with respect to $C\left(t_{j}, \tau_{j}\right)$ of the maximand on the right hand side of (A.8) must be non-negative. Therefore, differentiation of this maximand with respect to $C\left(t_{j}, \tau_{j}\right)$ yields

$$
\left(1-\frac{\theta_{1}(1-\alpha)}{e^{\omega \tau_{j}} h\left(\tau_{j}\right)}\right)\left[h\left(\tau_{j}\right)\right]^{\alpha}\left[C\left(t_{j}, \tau_{j}\right)\right]^{-\alpha}-e^{r_{L} \tau_{j}} \beta^{\tau_{j}} E_{t_{j}}\left\{V_{X}\left(e^{r_{L} \tau_{j}}\left(X_{t_{j}}-C\left(t_{j}, \tau_{j}\right)\right), R\left(\tau_{j}\right) S_{t_{j}}\right)\right\} \geq 0 .
$$

Since $V_{X}()>0,\left[h\left(\tau_{j}\right)\right]^{\alpha}\left[C\left(t_{j}, \tau_{j}\right)\right]^{-\alpha}>0$, and $e^{r_{L} \tau_{j}} \beta^{\tau_{j}}>0$, equation (A.11) implies that

$$
1-\frac{\theta_{1}(1-\alpha)}{e^{\omega \tau_{j}^{*} h}\left(\tau_{j}^{*}\right)}>0,
$$

where $\phi_{j}^{*}$ and $\tau_{j}^{*}$ are the values of $\phi_{j}$ and $\tau_{j}$ that maximize the restrictive value function. Now we consider the cases in which $\alpha<1$ and $\alpha>1$ separately.

Case I: $\alpha<1$. When $\alpha<1$, equation (A.12) implies a lower bound on $\tau^{*}$. Use the definition of $h\left(\tau_{j}\right)$ in equation (15) to rewrite equation (A.12) as

$$
\tau_{j}^{*}>\bar{\tau} \equiv \frac{1}{\omega} \ln \left(1+\omega \theta_{1}(1-\alpha)\right)>0 .
$$

Equation (13) implies that

$$
c_{t_{j}^{+}}=\frac{C\left(t_{j}, \tau_{j}\right)}{h\left(\tau_{j}\right)}<\frac{X_{t_{j}}}{h(\bar{\tau})}
$$

where the inequality follows from the constraint $C\left(t_{j}, \tau_{j}\right) \leq X_{t_{j}}$ and the facts that $h\left(\tau_{j}\right)$ is strictly increasing in $\tau_{j}$ and $\tau_{j}^{*}>\bar{\tau}$. Equation (A.14) implies $\lim _{X_{t_{j}} \rightarrow 0} c_{t_{j}^{+}}=0$. Therefore, taking the 
limits of both sides of equation (A.10) as $X_{t_{j}} \rightarrow 0$, and using the facts that $0 \leq C\left(t_{j}, \tau_{j}\right) \leq X_{t_{j}}$, $\tau_{j}^{*}>\bar{\tau}>0$ and $\lim _{X_{t_{j}} \rightarrow 0}\left(X_{t_{j}}-C\left(t_{j}, \tau_{j}\right)\right)=0$, implies

$\lim _{X_{t_{j}} \rightarrow 0} \widetilde{V}\left(X_{t_{j}}, S_{t_{j}}\right)=\lim _{X_{t_{j}} \rightarrow 0} \beta^{\tau_{j}} E_{t_{j}}\left\{V\left(0, R\left(\tau_{j}\right) S_{t_{j}}\right)\right\}=\lim _{X_{t_{j}} \rightarrow 0} \beta^{\tau_{j}^{*}} E_{t_{j}}\left\{\left[R\left(\tau_{j}^{*}\right)\right]^{1-\alpha}\right\} \frac{1}{1-\alpha} S_{t_{j}}^{1-\alpha} v(0)$

Use equation (9), the definition $\beta^{\tau_{j}} \equiv e^{-\rho \tau_{j}}$, and the fact that $\tau^{*}>\bar{\tau}$ to obtain

$$
\lim _{X_{t_{j}} \rightarrow 0} \tilde{V}\left(X_{t_{j}}, S_{t_{j}}\right)<\frac{1}{1-\alpha} S_{t_{j}}^{1-\alpha} v(0)=V\left(0, S_{t_{j}}\right) .
$$

Case II: $\alpha>1$. In the case with $\alpha>1, c_{t_{j}^{+}}$does not go to zero as $X_{t_{j}}$ approaches 0 , because the instantaneous flow of utility would be unboundedly negative. Thus, $\bar{c} \equiv \lim _{X_{t_{j}} \rightarrow 0} c_{t_{j}^{+}}>0$. Hence, equation (13) implies that $\lim _{X_{t_{j}} \rightarrow 0} \tau_{j}=0$. We now that show that $\bar{c}<\infty$, i.e., the consumption flow $c_{t_{j}^{+}}$approaches a finite limit. To see this, define $\Psi\left(\tau_{j}\right)$ as

$$
\Psi\left(\tau_{j}\right) \equiv \frac{h^{\prime}\left(\tau_{j}\right)}{h(\tau)}\left(\alpha+\frac{\theta_{1}(1-\alpha)}{h\left(\tau_{j}\right)-\theta_{1}(1-\alpha) e^{-\omega \tau_{j}}}\right)
$$

and note that the first order condition of (A.8) with respect to $\tau_{j}$ can be expressed as ${ }^{22}$

$\Psi\left(\tau_{j}\right)\left(1-\frac{\theta_{1}(1-\alpha)}{e^{\omega \tau_{j}} h\left(\tau_{j}\right)}\right) \frac{1}{1-\alpha}\left[h\left(\tau_{j}\right)\right]^{\alpha}\left[C\left(t_{j}, \tau_{j}\right)\right]^{1-\alpha}=-\frac{d}{d \tau_{j}}\left[\beta^{\tau_{j}} E_{t_{j}}\left\{V\left(e^{r_{L} \tau_{j}}\left(X_{t_{j}}-C\left(t_{j}, \tau_{j}\right)\right), R\left(\tau_{j}\right) S_{t_{j}}\right)\right\}\right]$.

As $X_{t_{j}} \rightarrow 0$, equation (22) implies that the right hand side of (A.18) approaches $-\frac{1}{1-\alpha} v(0) S_{t_{j}}^{1-\alpha} \frac{d}{d \tau_{j}}\left[\beta^{\tau_{j}} E_{t_{j}} R^{1-\alpha}\left(\tau_{j}\right)\right]$, which is finite. Hence, the left hand side of (A.18) must also approach a finite limit. Now suppose (counterfactually) that $\lim _{X_{t_{j}} \rightarrow 0} c_{t_{j}^{+}}=\infty$, and re-write the left hand side of (A.18) as $\left\{h\left(\tau_{j}\right) \Psi\left(\tau_{j}\right) \frac{1}{1-\alpha}\right\} \times\left\{\left(1-\frac{\theta_{1}(1-\alpha)}{e^{\omega \tau_{j}} h\left(\tau_{j}\right)}\right)\left[\frac{C\left(t_{j}, \tau_{j}\right)}{h\left(\tau_{j}\right)}\right]^{-\alpha}\right\} \times\left(\frac{C\left(t_{j}, \tau_{j}\right)}{h\left(\tau_{j}\right)}\right)$. By equations (A.17) and (15), $\lim _{X_{t_{j}} \rightarrow 0}\left\{h\left(\tau_{j}\right) \Psi\left(\tau_{j}\right) \frac{1}{1-\alpha}\right\}=\lim _{\tau_{j} \rightarrow 0}\left\{h\left(\tau_{j}\right) \Psi\left(\tau_{j}\right) \frac{1}{1-\alpha}\right\}=-1$. Equation (A.11) implies that $\lim _{X_{t_{j}} \rightarrow 0}\left\{\left(1-\frac{\theta_{1}(1-\alpha)}{e^{\omega \tau_{j}} h\left(\tau_{j}\right)}\right)\left[\frac{C\left(t_{j}, \tau_{j}\right)}{h\left(\tau_{j}\right)}\right]^{-\alpha}\right\}>0$, and the (counterfactual) assumption that $\lim _{X_{t_{j}} \rightarrow 0} c_{t_{j}^{+}}=\infty$ implies that $\lim _{X_{t_{j}} \rightarrow 0} \frac{C\left(t_{j}, \tau_{j}\right)}{h\left(\tau_{j}\right)}=\infty$ by equation (13). Hence, the left hand side of (A.18) approaches $-\infty$, so that equation (A.18) cannot hold. Therefore, $\lim _{X_{t_{j}} \rightarrow 0} c_{t_{j}^{+}}=\bar{c}<\infty$.

Now, taking the limits of both sides of equation (A.10) as $X_{t_{j}} \rightarrow 0$, and using the facts that $0 \leq C\left(t_{j}, \tau_{j}\right) \leq X_{t_{j}}$ and $\lim _{\tau_{j} \rightarrow 0} R\left(\tau_{j}\right)=1$, implies

$$
\lim _{X_{t_{j}} \rightarrow 0} \widetilde{V}\left(X_{t_{j}}, S_{t_{j}}\right)=-\theta_{1} \bar{c}_{t_{j}^{+}}^{1-\alpha}+V\left(0, S_{t_{j}}\right)<V\left(0, S_{t_{j}}\right) .
$$

Proof of $\boldsymbol{\omega}_{1}>\mathbf{0} . \quad$ Since $\lim _{x_{t_{j}} \rightarrow 0} \frac{1}{1-\alpha} \widetilde{v}\left(x_{t_{j}}\right)<\frac{1}{1-\alpha} v(0), \exists \bar{x}>0$ s.t. $\frac{1}{1-\alpha} \widetilde{v}(x)<\frac{1}{1-\alpha} v(0) \leq$ $\frac{1}{1-\alpha} v(x) \forall x \in[0, \bar{x}]$. Therefore, $\omega_{1} \geq \bar{x}>0$. 
Proof of $\pi_{2} \geq \pi_{1}$. To prove that $\pi_{2} \geq \pi_{1}$, suppose the contrary, i.e., that $\pi_{1}>\pi_{2}$, and consider three points $\left(X_{A}, S_{A}\right),\left(X_{B}, S_{B}\right)$, and $\left(X_{C}, S_{C}\right)$, where $X_{A}=\pi_{1} S_{A},\left(X_{B}, S_{B}\right)=$ $\left(\pi_{1} S_{A}-\left(1-\psi_{s}\right) z^{*}, S_{A}+z^{*}\right)$ where $z^{*} \equiv \frac{\pi_{1}-\pi_{2}}{\pi_{2}+1-\psi_{s}} S_{A}$, which implies $X_{B}=\pi_{2} S_{B},\left(X_{C}, S_{C}\right)=$ $\left(\pi_{2} S_{B}+\left(1+\psi_{b}\right) z^{* *}, S_{B}-z^{* *}\right)$ where $z^{* *} \equiv \frac{\pi_{1}-\pi_{2}}{\pi_{1}+1+\psi_{b}} S_{B}$, which implies $X_{C}=\pi_{1} S_{C}$. The definition of $\pi_{1}$ implies that $V\left(X_{A}, S_{A}\right) \geq V\left(X_{B}, S_{B}\right)$ and the definition of $\pi_{2}$ implies that $V\left(X_{B}, S_{B}\right) \geq$ $V\left(X_{C}, S_{C}\right)$ so that $V\left(X_{A}, S_{A}\right) \geq V\left(X_{C}, S_{C}\right)$. But $S_{C}=S_{B}-z^{* *}=S_{B}-\frac{\pi_{1}-\pi_{2}}{\pi_{1}+1+\psi_{b}} S_{B}=\frac{\pi_{2}+1+\psi_{b}}{\pi_{1}+1+\psi_{b}} S_{B}$ $=\frac{\pi_{2}+1+\psi_{b}}{\pi_{1}+1+\psi_{b}} \frac{\pi_{1}+1-\psi_{s}}{\pi_{2}+1-\psi_{s}} S_{A}=\left(\frac{\left(\pi_{1}-\pi_{2}\right)\left(\psi_{s}+\psi_{b}\right)}{\left(\pi_{1}+1+\psi_{b}\right)\left(\pi_{2}+1-\psi_{s}\right)}+1\right) S_{A}>S_{A}$, since $\psi_{s}+\psi_{b}>0$. Therefore, since $X_{C}=\pi_{1} S_{C}$ and $X_{A}=\pi_{1} S_{A}$, we have $X_{C}>X_{A}$. Hence, since $V(X, S)$ is strictly increasing in $X$ and $S$, we have $V\left(X_{C}, S_{C}\right)>V\left(X_{A}, S_{A}\right)$, which contradicts the earlier statement that $V\left(X_{A}, S_{A}\right) \geq V\left(X_{C}, S_{C}\right)$.

Proof of $\omega_{1} \leq \pi_{1}$. We will prove this statement using a geometric argument to show that $\omega_{1}>\pi_{1}$ leads to a contradiction. We consider three cases: $\theta_{S}<\theta_{X}, \theta_{S}>\theta_{X}$, and $\theta_{S}=\theta_{X}$.

Suppose that $\omega_{1}>\pi_{1}$ and consider the case in which $\theta_{S}<\theta_{X}$, so that in Figure 2(a) the line through points $B, C$, and $E$, which has slope $-\left(1-\psi_{s}\right) \frac{1-\theta_{S}}{1-\theta_{X}}$, is steeper than the line through points $C$ and $D$, which has slope $-\left(1-\psi_{s}\right)$. Statement 2c of Proposition 1 implies that for values of $x \equiv \frac{X}{S}$ less than $\omega_{1}$, indifference curves of the value function are straight lines with slope - $\left(1-\psi_{s}\right) \frac{1-\theta_{S}}{1-\theta_{X}}$. Therefore, $V(B)=V(C)=V(E)$, where the notation $V(J)$ indicates the value of the value function evaluated at point $J$. The definition of $\pi_{1}$ implies that $V(C) \geq V(D)$. Therefore, $V(E) \geq V(D)$, which contradicts strict monotonicity of the value function since both $X$ and $S$ are larger at point $D$ than at point $E$. Therefore, $\omega_{1} \leq \pi_{1}$ if $\theta_{S}<\theta_{X}$.

Suppose that $\omega_{1}>\pi_{1}$ and consider the case in which $\theta_{S}>\theta_{X}$, so that in Figure 2(b) the line through points $D$ and $E$, which has slope $-\left(1-\psi_{s}\right) \frac{1-\theta_{S}}{1-\theta_{X}}$, is less steep than the line through points $C$ and $E$, which has slope $-\left(1-\psi_{s}\right)$. Statement 2c of Proposition 1 implies that the line from point $D$ through point $E$ is an indifference curve and all points on this indifference curve are preferred to all points below and to the left of the indifference curve for which $x<\omega_{1}$. In particular, point $E$ is preferred to all points below point $E$ along the line through points $E$ and $C$. Since the value of $x$ at point $E$ is higher than $\pi_{1}$, the fact that the value function evaluated at point $E$ is greater than the value function, and hence greater than the restricted value function, evaluated at all points below point $E$ with slope $-\left(1-\psi_{s}\right)$ contradicts the definition of $\pi_{1}$. Therefore, $\omega_{1} \leq \pi_{1}$ if $\theta_{S}>\theta_{X}$.

Suppose that $\omega_{1}>\pi_{1}$ and consider the case in which $\theta_{S}=\theta_{X}$, so that in Figure 2(c) the slope of the line through points $C$ and $E$ is $-\left(1-\psi_{s}\right) \frac{1-\theta_{S}}{1-\theta_{X}}=-\left(1-\psi_{s}\right)$. Statement 2c of Proposition 1 implies that for values of $x \equiv \frac{X}{S}<\omega_{1}$, indifference curves of the value function are straight lines with slope $-\left(1-\psi_{s}\right) \frac{1-\theta_{S}}{1-\theta_{X}}$ so points $E$ and $C$ are on the same indifference curve. Indeed, point $E$ yields the same value of the value function as all points below point $E$ on the line through points $E$ and $C$. That is, for any point $J$ below point $E$ along the line through points $E$ and $C$ with $X \geq 0, V(E)=V(J)$. Since $x<\omega_{1}$ at point $J$, the definition of $\omega_{1}$ implies that $V(J)>\widetilde{V}(J)$. 


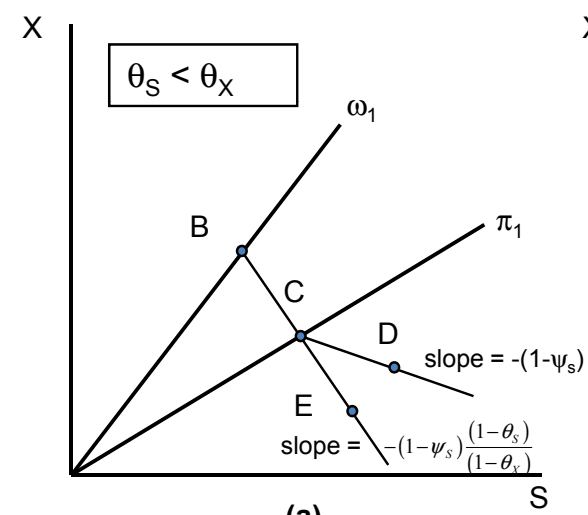

(a)

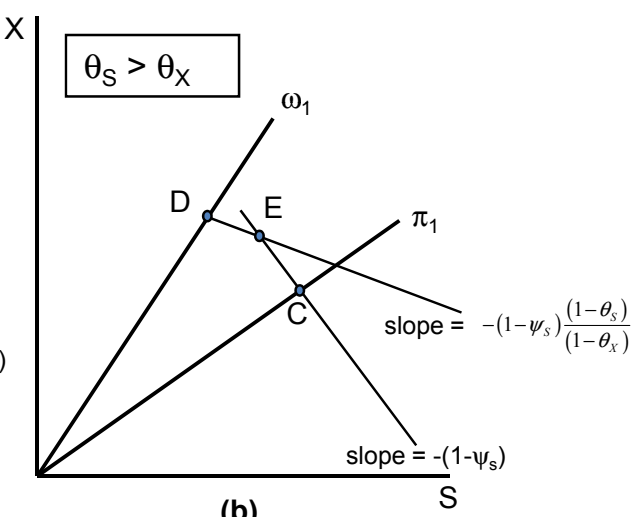

(b)

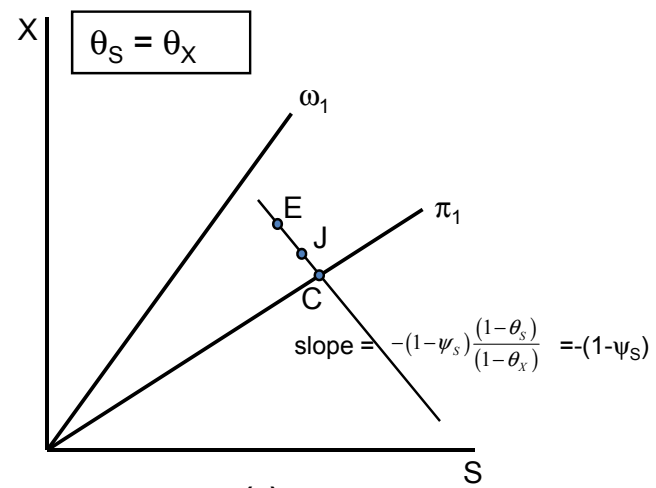

(c)

FiguRE 2:

Therefore, $V(E)=V(J)>\widetilde{V}(J)$. Since $x>\pi_{1}$ at point $E$, the facts that for arbitrary point $J$ we have $V(E)=V(J)$ and $V(E)>\widetilde{V}(J)$ contradict the definition of $\pi_{1}$. Therefore, $\omega_{1} \leq \pi_{1}$ if $\theta_{S}=\theta_{X}$.

Putting together the cases in which $\theta_{S}<\theta_{X}, \theta_{S}>\theta_{X}$, and $\theta_{S}=\theta_{X}$, we have proved that $\omega_{1} \leq \pi_{1}$.

Proof of $\omega_{2} \geq \pi_{2}$. Use a set of arguments similar to the proof that $\omega_{1} \leq \pi_{1}$.

Proof of $\omega_{2}<\infty$. We will prove that $\omega_{2}$ is finite by showing that if the investment portfolio has zero value on an observation date, the consumer will use some of the liquid assets in the transactions account to buy assets for the investment portfolio. We use proof by contradiction. That is, suppose that time 0 is an observation date, and that at this observation date, the transactions account has a balance $X_{0}>0$ and the investment portfolio has a zero balance so that $S_{0}=0$ and $x_{0}$ is infinite. Suppose that whenever the investment portfolio has zero value on an observation date, the consumer does not transfer any assets to the investment portfolio. Then the consumer will simply consume from the transactions account over the infinite future, never incurring any observation costs or transactions costs. In this case, with the values of the variables denoted with 
asterisks, $c_{0^{+}}^{*}=\frac{X_{0}}{h(\infty)}=\omega X_{0}, c_{t}^{*}=\exp \left(-\frac{\rho-r_{L}}{\alpha} t\right) c_{0^{+}}^{*}=\omega X_{t}^{*}$, so $X_{t}^{*}=\exp \left(-\frac{\rho-r_{L}}{\alpha} t\right) X_{0}$. Equation (12) implies that lifetime utility is

$$
U^{*}=\frac{1}{1-\alpha}[h(\infty)]^{\alpha} X_{0}^{1-\alpha}=\frac{1}{1-\alpha} \omega^{-\alpha} X_{0}^{1-\alpha}
$$

Now consider an alternative feasible path that sets $c_{t}=c_{t}^{*}$ for $0<t \leq T$ and at time $0^{+}$ transfers to the investment portfolio any liquid assets in the transactions account that will not be needed to finance consumption until time $T$. Under this alternative policy, the present value of consumption up to date $T$ is $h(T) c_{0^{+}}^{*}=h(T) \omega X_{0}$, so

$$
X_{0^{+}}=h(T) \omega X_{0} .
$$

The consumer uses $\left(1-\theta_{X}-\omega h(T)\right) X_{0}$ liquid assets to purchase assets in the investment portfolio. After paying the transactions cost,

$$
S_{0^{+}}=\frac{1-\theta_{X}-\omega h(T)}{1+\psi_{b}} X_{0}
$$

Suppose that the consumer invests the investment portfolio entirely in the riskless bond. At time $T$, the transactions account has a zero balance, and the investment portfolio is worth $S_{T}=$ $\exp \left(r_{f} T\right) \frac{1-\theta_{X}-\omega h(T)}{1+\psi_{b}} X_{0}$. The consumer converts the entire investment portfolio to the liquid asset in the transactions account, so that after paying the transactions costs, the balance in the transactions account is

$$
X_{T^{+}}=\left(1-\theta_{S}\right) \frac{1-\psi_{s}}{1+\psi_{b}} \exp \left(r_{f} T\right)\left[1-\theta_{X}-\omega h(T)\right] X_{0} .
$$

Define $P \equiv \frac{X_{T^{+}}}{X_{T}^{*}}$ as the ratio of the transactions account balance at time $T^{+}$under this alternative policy to the transactions account balance under the initial policy. Use equation (A.22) and $X_{T}^{*}=\exp \left(-\frac{\rho-r_{L}}{\alpha} T\right) X_{0}$, along with $\omega \equiv \frac{\rho-(1-\alpha) r_{L}}{\alpha}$, to obtain

$$
P \equiv \frac{X_{T^{+}}}{X_{T}^{*}}=\left(1-\theta_{S}\right) \frac{1-\psi_{s}}{1+\psi_{b}} F(T),
$$

where

$$
F(T) \equiv \exp \left[\left(r_{f}-r_{L}\right) T\right]\left[1-\theta_{X} \exp (\omega T)\right] .
$$

Now choose $T$ to maximize $F(T)$. Differentiate $F(T)$ and set the derivative equal to zero to obtain

$$
\exp (-\omega T)=\left(1+\frac{\omega}{r_{f}-r_{L}}\right) \theta_{X}<1,
$$

where the inequality follows from the assumption that $\theta_{X}<\overline{\theta_{X}}$ in equation (29) and the fact that $\frac{\omega}{r_{f}-r_{L}}>0$. Use equation (A.25) to evaluate $F(T)$ to obtain

$$
F(T)=\left(1+\frac{\omega}{r_{f}-r_{L}}\right)^{-1-\frac{r_{f}-r_{L}}{\omega}} \frac{\omega}{r_{f}-r_{L}} \theta_{X}^{-\frac{r_{f}-r_{L}}{\omega}} .
$$


Use equation (A.25) and the definition of $h(T)$ to obtain

$$
\omega h(T)=1-\left(1+\frac{\omega}{r_{f}-r_{L}}\right) \theta_{X}
$$

The present value of lifetime utility under the alternative plan is

$$
U=\left(1-\frac{\theta_{1}(1-\alpha)}{e^{\omega T} h(T)}\right) \frac{1}{1-\alpha}[h(T)]^{\alpha}\left[X_{0^{+}}\right]^{1-\alpha}+\exp (-\rho T) \frac{1}{1-\alpha}[h(\infty)]^{\alpha}\left[X_{T^{+}}\right]^{1-\alpha}
$$

Substitute equations (A.20) and (A.22) into equation (A.28) and use the facts that $h(\infty)=\frac{1}{\omega}$ and $1-\omega h(T)=\exp (-\omega T)$, and the definitions of $F(T)$ and $\omega$ to obtain

$$
\begin{aligned}
U= & \left(1-\frac{\theta_{1}(1-\alpha)}{e^{\omega T} h(T)}\right) \frac{1}{1-\alpha} h(T)\left[\omega X_{0}\right]^{1-\alpha} \\
& +\exp (-\rho T) \frac{1}{1-\alpha}\left[\frac{1}{\omega}\right]^{\alpha}\left[\left(1-\theta_{S}\right) \frac{1-\psi_{s}}{1+\psi_{b}} F(T) \exp \left(-\frac{\rho-r_{L}}{\alpha} T\right) X_{0}\right]^{1-\alpha} .
\end{aligned}
$$

Now divide the utility under the alternative plan in equation (A.29) by utility under the initial plan in equation (A.19) and use the definition of $\omega$ to obtain

$$
\frac{U}{U^{*}}=\omega h(T)-\theta_{1}(1-\alpha) \omega \exp (-\omega T)+\exp (-\omega T)\left[\left(1-\theta_{S}\right) \frac{1-\psi_{s}}{1+\psi_{b}} F(T)\right]^{1-\alpha} .
$$

Use the fact that $\omega h(T)=1-\exp (-\omega T)$ to rewrite equation (A.30) as

$$
\frac{U}{U^{*}}=1+\left(\left[\left(1-\theta_{S}\right) \frac{1-\psi_{s}}{1+\psi_{b}} F(T)\right]^{1-\alpha}-1-\theta_{1}(1-\alpha) \omega\right) \exp (-\omega T) .
$$

If $\alpha<1$, utility under the alternative plan, $U$, will exceed $U^{*}$ if $\frac{U}{U^{*}}>1$; if $\alpha>1$, utility under the alternative plan, $U$, will exceed $U^{*}$ if $\frac{U}{U^{*}}<1$. A sufficient condition for $U$ to exceed $U^{*}$, regardless of whether $\alpha$ is less than or greater than one, is

$$
F(T)>\left[\left(1-\theta_{S}\right) \frac{1-\psi_{s}}{1+\psi_{b}}\right]^{-1}\left[1+\theta_{1}(1-\alpha) \omega\right]^{\frac{1}{1-\alpha}}
$$

Since $F(T)$ in equation (A.26) is proportional to $\theta_{X}^{-\frac{r_{f}-r_{L}}{\omega}}$, the condition in equation (A.32) will be satisfied if $\theta_{X} \geq 0$ is sufficiently small. Specifically, this condition will be satisfied if

$$
\theta_{X}<\left(1+\frac{\omega}{r_{f}-r_{L}}\right)^{-1}\left(\frac{r_{f}-r_{L}}{\omega}+1\right)^{-\frac{\omega}{r_{f}-r_{L}}}\left[\left(1-\theta_{S}\right) \frac{1-\psi_{s}}{1+\psi_{b}}\right]^{\frac{\omega}{r_{f}-r_{L}}}\left[1+\theta_{1}(1-\alpha) \omega\right]^{\frac{-1}{1-\alpha} \frac{\omega}{r_{f}-r_{L}}},
$$


which is equivalent to $\theta_{X}<\overline{\theta_{X}}$ in equation (29). Since $\theta_{X}<\overline{\theta_{X}}$, the original plan, in which the consumer does not buy any assets in the investment portfolio, is not optimal.

The proof of statement 1 is now complete

Proof of statement 3. Suppose that on observation date $t_{j}$ the consumer has $\left(X_{t_{j}}, S_{t_{j}}\right)=$ $\left(\omega_{1} S_{t_{j}}, S_{t_{j}}\right)$ so that $x_{t_{j}}=\omega_{1}$. The proof of statement $2 \mathrm{~b}$ implies that if the consumer sells assets from the investment portfolio, he will choose $\left(X_{t_{j}^{+}}, S_{t_{j}^{+}}\right)=\left(\pi_{1} S_{t_{j}^{+}}, S_{t_{j}^{+}}\right)$so that $x_{t_{j}^{+}}=\pi_{1} \geq \omega_{1}$, and equation (7) implies that

$$
\frac{S_{t_{j}^{+}}}{S_{t_{j}}}=\frac{\left(1-\theta_{X}\right) \omega_{1}+\left(1-\theta_{S}\right)\left(1-\psi_{s}\right)}{\pi_{1}+1-\psi_{s}} .
$$

The value-matching condition states that the consumer is indifferent between the initial allocation with $x_{t_{j}}=\omega_{1}$ and the new allocation with $x_{t_{j}^{+}}=\pi_{1}$ so that

$$
V\left(\omega_{1} S_{t_{j}}, S_{t_{j}}\right)=V\left(\pi_{1} S_{t_{j}^{+}}, S_{t_{j}^{+}}\right) .
$$

Use equation (22), which is based on the homogeneity of the value function, to obtain

$$
S_{t_{j}}^{1-\alpha} v\left(\omega_{1}\right)=S_{t_{j}^{+}}^{1-\alpha} v\left(\pi_{1}\right) .
$$

Divide both sides of equation (A.36) by $S_{t_{j}}^{1-\alpha}$ and use equation (A.34) to obtain

$$
v\left(\omega_{1}\right)=\left[\frac{\left(1-\theta_{X}\right) \omega_{1}+\left(1-\theta_{S}\right)\left(1-\psi_{s}\right)}{\pi_{1}+1-\psi_{s}}\right]^{1-\alpha} v\left(\pi_{1}\right) .
$$

Observe from equation (A.37) that if $\theta_{X}=\theta_{S}=0$, then $\omega_{1}=\pi_{1}$. In this case, $\pi_{1}$ is both a trigger value and a return value. That is, if $x_{t_{j}}<\pi_{1}$ on observation date $t_{j}$, the consumer will sell assets from the investment portfolio to make $x_{t_{j}^{+}}=\pi_{1}$.

Proof of statements 4 and 5. The proof of statement 4 follows the proof of statement 2, and the proof of statement 5 follows the proof of statement 3 .

The proof of Proposition 1 is now complete.

Proof of Proposition 2. Proof by contradiction. Since $\theta_{1}>0$, the consumer will not continuously observe the value of the investment portfolio. That is $\tau_{j}>0$. If $x_{t_{j}}<\omega_{1}$ on an observation date $t_{j}$, then the consumer sells assets from the investment portfolio, increasing $X_{t_{j}}$ and decreasing $S_{t_{j}}$ until $x_{t_{j}^{+}}=\pi_{1}$. The consumer chooses $\tau_{j}$ and $C\left(t_{j}, \tau_{j}\right)$, which is the present value of consumption between times $t_{j}^{+}$and $t_{j+1}=t_{j}+\tau_{j}$. Suppose that the consumer chooses $C\left(t_{j}, \tau_{j}\right)<X_{t_{j}^{+}}$, which implies $X_{t_{j}+1}=\left[X_{t_{j}^{+}}-C\left(t_{j}, \tau_{j}\right)\right] e^{r_{L} \tau_{j}}>0$. We will show that there exists a deviation from this choice that will increase the consumer's expected lifetime utility, and hence $X_{t_{j+1}}>0$ cannot be optimal.

Consider a deviation in which the consumer reduces the amount of assets that she sells from the investment portfolio at time $t_{j}^{+}$by $\frac{X_{t_{j}^{+}}-C\left(t_{j}, \tau_{j}\right)}{1-\psi_{s}}=\frac{e^{-r_{L} \tau_{j}} X_{t_{j}+1}}{1-\psi_{s}}$ and invests this amount in the 
riskless bond. With this deviation, the value of the investment portfolio at time $t_{j+1}$ will exceed its value under the original policy by $\frac{X_{t_{j+1}}}{1-\psi_{s}} e^{\left(r_{f}-r_{L}\right) \tau_{j}}$ and the transactions account will have a zero balance at time $t_{j+1}$.

The deviation from the original path will take one of two forms at time $t_{j+1}^{+}$, depending on whether the consumer would transfer assets under the original plan at time $t_{j+1}^{+}$. First, consider the case in which the consumer transfers assets at time $t_{j+1}^{+}$. In this case, the consumer can sell the additional $\frac{X_{t_{j+1}}}{1-\psi_{s}} e^{\left(r_{f}-r_{L}\right) \tau_{j}}$ in the investment portfolio to obtain additional liquid assets worth $X_{t_{j+1}} e^{\left(r_{f}-r_{L}\right) \tau_{j}}$. Of course, this transfer of assets at time $t_{j+1}$ will incur a fixed cost equal to $\theta_{S}\left[S_{t_{j}+1}+\frac{X_{t_{j+1}}}{1-\psi_{s}} e^{\left(r_{f}-r_{L}\right) \tau_{j}}\right]$. However, since the consumer would have transferred assets under the original plan, she would have had to incur a fixed cost equal to $\theta_{X} X_{t_{j+1}}+\theta_{S} S_{t_{j+1}}$. Therefore the net gain of the deviation is $\left[X_{t_{j+1}} e^{\left(r_{f}-r_{L}\right) \tau_{j}}-X_{t_{j+1}}\right]-\left[\theta_{S}\left(S_{t_{j}+1}+\frac{X_{t_{j+1}}}{1-\psi_{s}} e^{\left(r_{f}-r_{L}\right) \tau_{j}}\right)-\left(\theta_{X} X_{t_{j+1}}+\theta_{S} S_{t_{j+1}}\right)\right]$ $=\left[\left(1-\frac{\theta_{S}}{1-\psi_{s}}\right) e^{\left(r_{f}-r_{L}\right) \tau_{j}}-1+\theta_{X}\right] X_{t_{j+1}} . \quad$ Since $\quad\left(r_{f}-r_{L}\right) \tau_{j}>0$, $\lim _{\max \left(\theta_{X}, \theta_{S}\right) \rightarrow 0}\left[\left(1-\frac{\theta_{S}}{1-\psi_{s}}\right) e^{\left(r_{f}-r_{L}\right) \tau_{j}}-1+\theta_{X}\right] X_{t_{j+1}}=\left(e^{\left(r_{f}-r_{L}\right) \tau_{j}}-1\right) X_{t_{j+1}}>0$, and the net gain of the deviation is positive.

Now consider the case in which the consumer would not make any transfers between investment portfolio and the transactions account at time $t_{j+1}^{+}$under the original policy, which implies that $\omega_{1} \leq x_{t_{j+1}} \leq \omega_{2}$. In this case, instead of selling the additional $\frac{X_{t_{j+1}}}{1-\psi_{s}} e^{\left(r_{f}-r_{L}\right) \tau_{j}}$ assets in the investment portfolio, the consumer only sells $z \equiv\left(1-\theta_{S}\right)\left(S_{t_{j+1}}+\frac{X_{t_{j+1}}}{1-\psi_{s}} e^{\left(r_{f}-r_{L}\right) \tau_{j}}\right)-S_{t_{j+1}}$ from the investment portfolio at time $t_{j+1}^{+}$. We observe that if $\theta_{S}$ is small enough, then $z>0$. Moreover, by construction of $z$, the consumer has the same value of assets in the investment portfolio under both the original plan and the deviation, since $S_{t_{j+1}}=\left(1-\theta_{S}\right)\left(S_{t_{j+1}}+\frac{X_{t_{j+1}}}{1-\psi_{s}} e^{\left(r_{f}-r_{L}\right) \tau_{j}}\right)-z$. Hence, the consumer will prefer the deviation if $z\left(1-\psi_{s}\right)>X_{t_{j+1}^{+}}=X_{t_{j+1}}$. Using the definition of $z$, the consumer will prefer the deviation if $\left(1-\theta_{S}\right) X_{t_{j+1}} e^{\left(r_{f}-r_{L}\right) \tau_{j}}-\theta_{S}\left(1-\psi_{s}\right) S_{t_{j+1}}>X_{t_{j+1}}$, which is equivalent to $\left(1-\theta_{S}\right) e^{\left(r_{f}-r_{L}\right) \tau_{j}}-\frac{\theta_{S}\left(1-\psi_{s}\right)}{x_{t_{j+1}}}>1$. Since $\left(r_{f}-r_{L}\right) \tau_{j}>0$ and $x_{t_{j+1}} \geq \omega_{1}>0$, $\lim _{\theta_{S} \rightarrow 0}\left[\left(1-\theta_{S}\right) e^{\left(r_{f}-r_{L}\right) \tau_{j}}-\frac{\theta_{S}\left(1-\psi_{s}\right)}{x_{t_{j+1}}}\right]>1$. Therefore, for sufficiently small $\theta_{S}$, the consumer prefers the deviation.

We have shown that if $x_{t_{j}}<\omega_{1}$, then $x_{t_{j+1}}=0<\omega_{1}$. Therefore, $x_{t_{j+2}}=0$ and so on, ad infinitum.

Proof of Lemma 1. Lemma 4 states that the optimal value of $\phi_{j}$ is positive. Since $\tau_{j}>0$ as a consequence of the observation cost, there exists some $\delta>0$ such that between any two consecutive observation dates, $t_{j}$ and $t_{j+1}, \operatorname{Pr}\left\{e^{-r_{L} \tau_{j}} R\left(\tau_{j}\right)>\frac{\omega_{2}}{\omega_{1}}\right\} \geq \delta$. Therefore, since $x_{t_{j+1}} \equiv \frac{X_{t_{j+1}}}{S_{t_{j+1}}}=\frac{e^{r} L_{j} \tau_{j}}{R\left(\tau_{j}\right)} \frac{X_{t_{j}^{+}}-C\left(t_{j}, \tau_{j}\right)}{S_{t_{j}^{+}}}<\frac{e^{r_{L} \tau_{j}}}{R\left(\tau_{j}\right)} \frac{X_{t_{j}^{+}}}{S_{t_{j}^{+}}}=\frac{x_{t_{j}^{+}}}{e^{-r_{L} \tau_{j}} R\left(\tau_{j}\right)} \leq \frac{\omega_{2}}{e^{-r_{L} \tau_{j}} R\left(\tau_{j}\right)}$ (where the final inequality follows from Corollary 1), $\operatorname{Pr}\left\{x_{t_{j+1}}<\omega_{1}\right\} \geq \delta$. Let $t_{k} \geq t_{j}$ be the first observation date at which $x_{t_{k}}<\omega_{1}$. Then by Williams (1991), p. 233, Pr $\left\{t_{k}<\infty\right\}=1$ and $E\left\{t_{k}\right\}<\infty$.

Proof of Proposition 4. Suppose that $\psi_{s}=0$ and let $\left\{S_{t}^{*}\right\}_{t=t_{j}}^{t=\infty}$ be the path of the $S_{t}$ under 
the optimal policy starting from observation date $t_{j}$ when the consumer observes $X_{t_{j}}=0$ and $S_{t_{j}}=S_{t_{j}}^{*}$. Let $\tau^{*}$ be the constant optimal interval of time between consecutive observations so that observation date $t_{k}=t_{j}+(k-j) \tau^{*}$, for $k \geq j$. For any observation date $t_{k} \geq t_{j}$, the transactions account balance will be $X_{t_{k}}=0$, and immediately after each observation date the transactions account balance will be $X_{t_{k}^{+}}=X_{t_{k}^{+}}^{*} \equiv \pi_{1}(0) S_{t_{k}^{+}}^{*}$. Since $0=X_{t_{k+1}}^{*}=e^{r_{L} \tau^{*}}\left(X_{t_{k}^{+}}^{*}-C\left(t_{k}, \tau^{*}\right)\right)$, we have $C\left(t_{k}, \tau^{*}\right)=X_{t_{k}^{+}}^{*}$

Now let $\psi_{s}$ take an arbitrary admissible and suppose that the consumer continues to observe the value of the investment portfolio on dates $t_{k}=t_{j}+(k-j) \tau^{*}$, for $k \geq j$, and maintains the same path of $S_{t}$, i.e., that $S_{t}=S_{t}^{*}$ for $t \geq t_{j}$. Since the consumer will make the same transfers out of the investment portfolio as in the initial case with $\psi_{s}=0$, a feasible path of the transaction account balance immediately after each observation date would be $X_{t_{k}^{+}}=\left(1-\psi_{s}\right) X_{t_{k}^{+}}^{*}$, which supports a feasible path of consumption of $C\left(t_{k}, \tau^{*}\right)=\left(1-\psi_{s}\right) X_{t_{k}^{+}}^{*}$. Therefore, $V\left(0, S_{t_{j}} ; \psi_{s}\right) \geq$ $\left(1-\psi_{s}\right)^{1-\alpha} V\left(0, S_{t_{j}} ; 0\right)$.

A similar argument starting with an arbitrary admissible value of $\psi_{s}$ less than one implies $V\left(0, S_{t_{j}} ; 0\right) \geq\left(\frac{1}{1-\psi_{s}}\right)^{1-\alpha} V\left(0, S_{t_{j}} ; \psi_{s}\right)$. Therefore, $V\left(0, S_{t_{j}} ; \psi_{s}\right) \geq\left(1-\psi_{s}\right)^{1-\alpha} V\left(0, S_{t_{j}} ; 0\right) \geq$ $V\left(0, S_{t_{j}} ; \psi_{s}\right)$, which implies $V\left(0, S_{t_{j}} ; \psi_{s}\right)=\left(1-\psi_{s}\right)^{1-\alpha} V\left(0, S_{t_{j}} ; 0\right)$ (statement 1$)$. We showed that by maintaining the same observation dates when $\psi_{s}$ is positive as when $\psi_{s}=0$ allows a path of consumption that achieves $V\left(0, S_{t_{j}} ; \psi_{s}\right) \geq\left(1-\psi_{s}\right)^{1-\alpha} V\left(0, S_{t_{j}} ; 0\right)=V\left(0, S_{t_{j}} ; \psi_{s}\right)$. Similarly, by maintaining the same observation dates when $\psi_{s}=0$ as when $\psi_{s}$ is positive allows a path of consumption that achieves $V\left(0, S_{t_{j}} ; 0\right) \geq\left(\frac{1}{1-\psi_{s}}\right)^{1-\alpha} V\left(0, S_{t_{j}} ; \psi_{s}\right)=V\left(0, S_{t_{j}} ; 0\right)$. Therefore, we have proven statement 2. For any observation date $t_{k} \geq t_{j}, x_{t_{k}^{+}}=\pi_{1}\left(\psi_{s}\right)$. Therefore, $\pi_{1}\left(\psi_{s}\right)=\frac{X_{t_{k}^{+}}}{S_{t_{k}^{+}}}=\frac{\left(1-\psi_{s}\right) X_{t_{k}^{+}}^{*}}{S_{t_{k}^{+}}^{*}}=\left(1-\psi_{s}\right) \pi_{1}(0)$, which proves statement 3.

Proof of Proposition 5. At each observation date $t_{j}$ the consumer chooses the share $\phi_{j}$ of the investment portfolio to allocate to equity to maximize $E_{t_{j}}\left\{V\left(X_{t_{j+1}}, S_{t_{j+1}}\right)\right\}$ subject to the constraints $0 \leq \phi_{j} \leq 1$. Using equations (2) and (3), we can write the Lagrangian for this constrained maximization as

$$
\mathcal{L}_{j}=E_{t_{j}}\left\{V\left(X_{t_{j+1}}, \phi_{j} \frac{P_{t_{j+1}}}{P_{t_{j}}} S_{t_{j}^{+}}+\left(1-\phi_{j}\right) e^{r_{f} \tau_{j}} S_{t_{j}^{+}}\right)\right\}+\delta_{j} S_{t_{j}^{+}} \phi_{j}+\xi_{j} S_{t_{j}^{+}}\left(1-\phi_{j}\right)
$$

where $\delta_{j} S_{t_{j}^{+}} \geq 0$ is the Lagrange multiplier on the constraint $\phi_{j} \geq 0$ and $\xi_{j} S_{t_{j}^{+}} \geq 0$ is the Lagrange multiplier on the constraint $\phi_{j} \leq 1$. Differentiating the Lagrangian in equation (A.38) with respect to $\phi_{j}$, setting the derivative equal to zero, and then dividing both sides by $S_{t_{j}^{+}}$yields

$$
E_{t_{j}}\left\{V_{S}\left(X_{t_{j+1}}, S_{t_{j+1}}\right)\left(\frac{P_{t_{j+1}}}{P_{t_{j}}}-e^{r_{f} \tau_{j}}\right)\right\}=\xi_{j}-\delta_{j}
$$

Next, we prove the following lemma.

Lemma $4 \phi_{j}>0$ and $\delta_{j}=0$. 
Proof of Lemma 4. We will proceed by contradiction. Suppose that $\phi_{j}=0$, which implies that $\xi_{j}=0$ and that $S_{t_{j+1}}$ is known at time $t_{j}$. Therefore, equation (A.39) can be written as $V_{S}\left(X_{t_{j+1}}, S_{t_{j+1}}\right) E_{t_{j}}\left\{\left(\frac{P_{t_{j+1}}}{P_{t_{j}}}-e^{r_{f}} \tau_{j}\right)\right\}=-\delta_{j} \leq 0$, which is a contradiction because $V_{S}\left(X_{t_{j+1}}, S_{t_{j+1}}\right)>0$ and, by assumption, the expected equity premium, $E_{t_{j}}\left\{\left(\frac{P_{t_{j+1}}}{P_{t_{j}}}-e^{r_{f} \tau_{j}}\right)\right\}$, is positive. Therefore, $\phi_{j}$ must be positive, which implies $\delta_{j}=0$.

To replace the marginal valuation of the investment portfolio, $V_{S}\left(X_{t_{j+1}}, S_{t_{j+1}}\right)$, by a function of the marginal utility of consumption, first use the definition of the marginal rate of substitution $m\left(x_{t_{j+1}}\right)$ to obtain

$$
V_{S}\left(X_{t_{j+1}}, S_{t_{j+1}}\right)=m\left(x_{t_{j+1}}\right) V_{X}\left(X_{t_{j+1}}, S_{t_{j+1}}\right) .
$$

Then use the envelope theorem to obtain

$$
V_{X}\left(X_{t_{j+1}}, S_{t_{j+1}}\right)=\left[1-\left(\mathbf{1}_{\left\{y_{t_{j+1}}^{b}>0\right\}}+\mathbf{1}_{\left\{y_{t_{j+1}}^{s}<0\right\}}\right) \theta_{X}\right]\left(1-\frac{\theta_{1}(1-\alpha)}{e^{\omega \tau_{j} h\left(\tau_{j+1}\right)}}\right) U^{\prime}\left(C\left(t_{j+1}, \tau_{j+1}\right)\right)
$$

which implies that $V_{X}\left(X_{t_{j+1}}, S_{t_{j+1}}\right)$, the increase in expected lifetime utility made possible by a one-dollar increase in $X_{t_{j+1}}$, equals the increase in utility that would accompany an increase of $1-\left(\mathbf{1}_{\left\{y_{t_{j+1}}^{b}>0\right\}}+\mathbf{1}_{\left\{y_{t_{j+1}}^{s}<0\right\}}\right) \theta_{X}$ dollars in $C\left(t_{j+1}, \tau_{j+1}\right)$. That is, if consumer transfers assets between the investment portfolio and the transactions account at time $t_{j+1}^{+}$, a one-dollar increase in $X_{t_{j+1}}$ would allow $C\left(t_{j+1}, \tau_{j+1}\right)$ to increase by $1-\theta_{X}$ dollars; otherwise, $C\left(t_{j+1}, \tau_{j+1}\right)$ can increase by one dollar. Substitute equation (A.41) into equation (A.40) and use the expression for the marginal utility of consumption from equation (14) to obtain

$$
V_{S}\left(X_{t_{j+1}}, S_{t_{j+1}}\right)=m\left(x_{t_{j+1}}\right)\left[1-\left(\mathbf{1}_{\left\{y_{t_{j+1}}^{b}>0\right\}}+\mathbf{1}_{\left\{y_{t_{j+1}}^{s}<0\right\}}\right) \theta_{X}\right]\left(1-\frac{\theta_{1}(1-\alpha)}{e^{\omega \tau_{j}} h\left(\tau_{j+1}\right)}\right) c_{t_{j+1}^{+}}^{-\alpha} .
$$

Substituting the right hand side of equation (A.42) for $V_{S}\left(X_{t_{j+1}}, S_{t_{j+1}}\right)$ in equation (A.39) and using Lemma 4 to set $\delta_{j}=0$ yields

$$
E_{t_{j}}\left\{m\left(x_{t_{j+1}}\right)\left[1-\left(\mathbf{1}_{\left\{y_{t_{j+1}}^{b}>0\right\}}+\mathbf{1}_{\left\{y_{t_{j+1}}^{s}<0\right\}}\right) \theta_{X}\right]\left(1-\frac{\theta_{1}(1-\alpha)}{e^{\omega \tau_{j}} h\left(\tau_{j+1}\right)}\right) c_{t_{j+1}^{+}}^{-\alpha}\left(\frac{P_{t_{j+1}}}{P_{t_{j}}}-e^{r_{f} \tau_{j}}\right)\right\}=\xi_{j} .
$$

In standard models without observation costs and transfer costs, and without the constraints $0 \leq$ $\phi_{j} \leq 1$, the corresponding Euler equation, which is widely used in financial economics, is

$$
E_{t}\left\{c_{s}^{-\alpha}\left(\frac{P_{s}}{P_{t}}-e^{r_{f}(s-t)}\right)\right\}=0 \text { for } s>t .
$$

In general, the Euler equation in the presence of observation costs and transactions costs in equation (A.43) differs from the standard Euler equation in equation (A.44) in three ways: (1) the Euler equation in equation (A.43) contains the Lagrange multiplier on the constraint $\phi_{j} \leq$ 
1 but this Lagrange multiplier does not appear in the standard Euler equation; (2) the Euler equation in equation (A.43) contains the marginal rate of substitution $m\left(x_{t_{j+1}}\right)$, which is a random variable, but this marginal rate of substitution is absent (or implicitly equal to a constant) in the standard Euler equation; ${ }^{23}$ (3) the Euler equation in equation (A.43) contains the term 1 $\left(\mathbf{1}_{\left\{y_{t_{j+1}}^{b}>0\right\}}+\mathbf{1}_{\left\{y_{t_{j+1}}^{s}<0\right\}}\right) \theta_{X}$, which reflects the additional fixed transfer cost associated with having an additional dollar in the transactions account; (4) the Euler equation in equation (A.43) contains the term $1-\frac{\theta_{1}(1-\alpha)}{e^{\omega \tau_{j}} h\left(\tau_{j+1}\right)}$, which reflects the utility cost of the next observation; and (5) in the presence of observation costs, the Euler equation holds only for rates of return between observation dates, whereas the Euler equation in the standard case holds for rates of return between any arbitrary pair of dates because all dates are observation dates in the standard case. We show that in the long run in an interesting special case, the first four of these differences disappear. Before showing this result, we prove the following lemma.

Lemma 5 Suppose that $\theta_{X}$ and $\theta_{S}$ are sufficiently small, in the sense described in the proof of Proposition 2. If $x_{t_{j}} \leq \omega_{1}$, then (i) $\phi_{j}<1$ if $\alpha>\frac{\mu-r_{f}}{\sigma^{2}}$ and (ii) $\phi_{j}=1$ if $\alpha \leq \frac{\mu-r_{f}}{\sigma^{2}}$.

Proof of Lemma 5. Proposition 2 implies that if $x_{t_{j}} \leq \pi_{1}$, then $x_{t_{j+1}}=0$. The optimal value of $\phi_{j}, 0 \leq \phi_{j} \leq 1$, maximizes $E_{t_{j}}\left\{V\left(X_{t_{j+1}}, S_{t_{j+1}}\right)\right\}=\frac{1}{1-\alpha} E_{t_{j}}\left\{S_{t_{j+1}}^{1-\alpha} v(0)\right\}$, which is equivalent to maximizing $\varphi\left(\phi_{j} ; \alpha\right) \equiv \frac{1}{1-\alpha} E_{t_{j}}\left\{\left[\phi_{j} \frac{P_{t_{j}+\tau_{j}}}{P_{t_{j}}}+\left(1-\phi_{j}\right) e^{r_{f} \tau_{j}}\right]^{1-\alpha}\right\}$. Define $\alpha^{*}$ such that $\arg \max _{\phi_{j}} \varphi\left(\phi_{j} ; \alpha^{*}\right)=1$ and note that $\varphi^{\prime}\left(1 ; \alpha^{*}\right)=0$.

Differentiating the definition of $\varphi\left(\phi_{j} ; \alpha\right)$ with respect to $\phi_{j}$ and setting $\phi_{j}=1$ yields

$$
\varphi^{\prime}(1 ; \alpha)=E_{t_{j}}\left\{\left(\frac{P_{t_{j}+\tau_{j}}}{P_{t_{j}}}\right)^{1-\alpha}\right\}-e^{r_{f} \tau_{j}} E_{t_{j}}\left\{\left(\frac{P_{t_{j}+\tau_{j}}}{P_{t_{j}}}\right)^{-\alpha}\right\} .
$$

Use the fact that $\frac{P_{t_{j}+\tau_{j}}}{P_{t_{j}}}$ is lognormal to obtain

$$
\varphi^{\prime}(1 ; \alpha)=\exp \left[(1-\alpha)\left(\mu-\frac{1}{2} \alpha \sigma^{2}\right) \tau_{j}\right]-e^{r_{f} \tau_{j}} \exp \left[-\alpha\left(\mu+\frac{1}{2}(-\alpha-1) \sigma^{2}\right) \tau_{j}\right] .
$$

Further rearrangement yields

$$
\varphi^{\prime}(1 ; \alpha)=\exp \left[\left(-\alpha \mu+r_{f}-\frac{1}{2} \alpha(1-\alpha) \sigma^{2}\right) \tau_{j}\right] \times\left[\exp \left(\left(\mu-r_{f}\right) \tau_{j}\right)-\exp \left(\alpha \sigma^{2} \tau_{j}\right)\right],
$$

which implies that

$$
\varphi^{\prime}(1 ; \alpha) \lesseqgtr 0 \text { as } \alpha \gtreqless \alpha^{*} \equiv\left(\mu-r_{f}\right) / \sigma^{2} .
$$

Differentiate $\varphi\left(\phi_{j} ; \alpha\right)$ twice with respect to $\phi_{j}$ to obtain

$$
\varphi^{\prime \prime}\left(\phi_{j} ; \alpha\right)=-\alpha E_{t_{j}}\left\{\left(\phi_{j} \frac{P_{t_{j}+\tau_{j}}}{P_{t_{j}}}+\left(1-\phi_{j}\right) e^{r_{f} \tau_{j}}\right)^{-\alpha-1}\left(\frac{P_{t_{j}+\tau_{j}}}{P_{t_{j}}}-e^{r_{f} \tau_{j}}\right)^{2}\right\}<0
$$


which implies that $\varphi\left(\phi_{j} ; \alpha\right)$ is concave. If $\alpha>\alpha^{*}$, then $\varphi^{\prime}(1 ; \alpha)<0$, so the concavity of $\varphi\left(\phi_{j} ; \alpha\right)$ implies that the optimal value of $\phi_{j}$ is less than one and the Lagrange multiplier on the constraint $\phi_{j} \leq 1$ is $\xi_{j}=0$. If $\alpha \leq \alpha^{*}$, then $\varphi^{\prime}(1 ; \alpha) \geq 0$, so the concavity of $\varphi\left(\phi_{j} ; \alpha\right)$ implies that the optimal value of $\phi_{j}$ equals one. If $\alpha<\alpha^{*}$, the Lagrange multiplier on the constraint $\phi_{j} \leq 1$ is $\xi_{j}>0$.

Suppose that $\theta_{X}$ and $\theta_{S}$ are sufficiently small so that in the long run, the stochastic process for $x_{t_{j}}$ is absorbed at zero. Lemma 5 implies that if the coefficient of relative risk aversion $\alpha$ exceeds $\frac{\mu-r_{f}}{\sigma^{2}}$, then in the long run the constraint $\phi_{j} \leq 1$ does not bind, and hence $\xi_{j}=0$. In this case, the first of the five differences between the Euler equation in equation (A.43) and the standard Euler equation disappears. In addition, in the long run $x_{t_{j}}=0$ on each observation date $t_{j}$ so $(1) m\left(x_{t_{j}}\right)=\left(1-\psi_{s}\right) \frac{1-\theta_{S}}{1-\theta_{X}}$ on each observation date, (2) the consumer sells assets from the investment portfolio on each observation date so $1-\left(\mathbf{1}_{\left\{y_{t_{j+1}}^{b}>0\right\}}+\mathbf{1}_{\left\{y_{t_{j+1}}^{s}<0\right\}}\right) \theta_{X}=$ $1-\theta_{X}$ on each observation date, and (3) the time between consecutive observations is constant so $1-\frac{\theta_{1}(1-\alpha)}{e^{\omega \tau_{j}} h\left(\tau_{j+1}\right)}$ is constant. Using the fact that $\xi_{j}=0$ and dividing both sides of equation (A.43) by $\left(1-\psi_{s}\right)\left(1-\theta_{S}\right)\left(1-\frac{\theta_{1}(1-\alpha)}{e^{\omega \tau} h(\tau)}\right)$, proves proposition 5 . 


\section{Notes}

${ }^{1}$ Stokey (2009) presents a comprehensive analysis of issues related to inaction and infrequent adjustment.

${ }^{2}$ We call this cost the "observation cost," though it summarizes all costs associated with obtaining and processing the information necessary to choose consumption and the allocation of assets.

${ }^{3}$ Woodford (2008) applies the concept of rational inattention in the context of pricing behavior by firms.

${ }^{4}$ Reis (2006) develops and analyzes a model of optimal inattention for a consumer who faces a cost of observing additive income, such as labor income. In that model, the consumer can hold only a single riskless asset so there is no portfolio allocation problem.

${ }^{5}$ Gabaix and Laibson (2002) is very similar to Abel, Eberly, and Panageas (2007), except that Gabaix and Laibson model the cost of observing the stock market as a utility cost and Abel, Eberly, and Panageas model it as a resource cost. A more important difference, however, is that the formulation of this cost used by Abel, Eberly, and Panageas preserves the homogeneity of the value function in wealth, thereby permitting exact analytic solution of the value function. However, the formulation adopted by Gabaix and Laibson does not preserve this homogeneity. (In private correspondence, Gabaix and Laibson (November 25, 2006) clarify their observation cost by stating "that the utility cost is always $q w_{0} e^{-\rho t}$, not $q w_{t} e^{-\rho t}$," so that the cost is not proportional to the contemporaneous value of wealth.) Therefore, Gabaix and Laibson compute an approximate solution.

${ }^{6}$ Huang and Liu (2007) apply the concept of rational inattention to study the optimal portfolio decision of an investor who can obtain costly noisy signals about a state variable governing the expected growth rate of stock prices. Although the substantive topic of their application of rational inattention is very close to the topic of our current paper, the economic framework is quite different. Importantly, Huang and Liu do not impose a cash-in-advance constraint that requires consumption to be purchased with a liquid asset. As a consequence, they focus on the investment portfolio of risky equity and bonds, but do not have a transactions account, whereas our focus is on the transfers of assets between the investment portfolio and the transactions account. In addition, Huang and Liu do not include any costs of trading assets and they allow continuous observation of stock prices so that the investor continuously trades assets within the investment portfolio. However, our modeling of transfer costs and infrequent observation of stock prices leads to infrequent transfers of assets.

${ }^{7}$ Our earlier paper assumes that the investment portfolio is continuously re-balanced by a portfolio manager who, on each observation date, charges a fee proportional to the size of the portfolio (and thus the fee is not separately identifiable from an observation cost that is proportional to the size of the portfolio) whereas the current paper does not allow re-balancing of the investment portfolio between observation dates.

${ }^{8}$ Because the transactions account does not include any risky assets, the consumer continuously knows the value of $X_{t}$.

${ }^{9}$ The consumer does not observe any new information between time $t_{j}^{+}$and time $t_{j+1}$ and hence cannot adjust the portfolio in response to any news that arrives during this interval of inattention. It is possible that the consumer could decide at time $t_{j}^{+}$to exchange equity for bonds at some time(s) before $t_{j+1}$, but we do not consider this possibility in this paper. In Abel, Eberly, and Panageas (2007) a portfolio manager continuously rebalances equity and bonds in the investment portfolio.

${ }^{10}$ This interpretation of $\psi_{s}$ as a tax rate is most plausible if the consumer only withdraws money from the investment portfolio and never transfers assets into the investment portfolio. As we will see in Section 3, the long run is characterized by precisely this situation in which the consumer never transfers funds into the 
investment portfolio, if the fixed component of the transfer cost is sufficiently small.

${ }^{11}$ We assume that $\theta_{X}$ is very small so that if $X>0$ and $S=0$, the consumer will not be deterred from transferring at least some assets from the transactions account to the investment portfolio. We assume that $\psi_{s}+\theta_{S}<1$ to prevent assets from becoming "trapped" in the investment portfolio if the consumer were to try to sell assets from the investment portfolio at a time when $X=0$. If, instead, $\psi_{s}+\theta_{S}$ were greater than or equal to one, then an attempt to sell a dollar of assets from the investment portfolio would cost at least one dollar and the consumer would not receive any liquid assets as a result of this transaction.

${ }^{12}$ Duffie and Sun (1990) assume that on each observation date the consumer pays a portfolio management fee that is proportional to total wealth. In their model, optimal behavior implies that $X=0$ on each observation date, so the fixed transaction cost $\theta_{X} X+\theta_{S} S$ is simply $\theta_{S} S$; hence, they do not need to explicitly specify the value of $\theta_{X}$.

${ }^{13}$ As we will show, if $S_{t_{j}}=0$ on observation date $t_{j}$, the consumer will use some liquid assets from the transactions account to purchase assets in the investment portfolio, so $S_{t_{j}^{+}}>0$. Then on all subsequent observation dates, $S_{t_{j}}>0$.

${ }^{14}$ The optimal values of consumption during the interval of time from $t_{j}^{+}$to $t_{j+1}$ satisfy the condition that the product of the intertemporal marginal rate of substitution between times $t_{j}^{+}$and $s,\left(\frac{c_{s}}{c_{t_{j}^{+}}}\right)^{-\alpha} e^{-\rho\left(s-t_{j}^{+}\right)}$, and the gross rate of return between those times, $e^{r_{L}\left(s-t_{j}^{+}\right)}$, equals one, so that

$$
c_{s}=e^{-\frac{\rho-r_{L}}{\alpha}\left(s-t_{j}^{+}\right)} c_{t_{j}^{+}}, \text {for } t_{j}^{+} \leq s \leq t_{j+1} .
$$

Substituting $c_{s}$ from equation $(*)$ into equation (10) in the text yields

$$
C\left(t_{j}, \tau_{j}\right)=h\left(\tau_{j}\right) c_{t_{j}^{+}}
$$

where $h\left(\tau_{j}\right)$ is defined in equation (15) in the text. Equations $\left(^{*}\right)$ and $(* *)$ imply that

$$
c_{s}=\left[h\left(\tau_{j}\right)\right]^{-1} e^{-\frac{\rho-r_{L}}{\alpha}\left(s-t_{j}^{+}\right)} C\left(t_{j}, \tau_{j}\right), \quad \text { for } t_{j}^{+} \leq s \leq t_{j+1} .
$$

Substituting equation (***) into equation (11), and using the definition of $h\left(\tau_{j}\right)$ in equation (15) yields $U\left(C\left(t_{j}, \tau_{j}\right)\right)=\frac{1}{1-\alpha}\left[h\left(\tau_{j}\right)\right]^{\alpha}\left[C\left(t_{j}, \tau_{j}\right)\right]^{1-\alpha}$, which, along with equation $(* *)$, implies that $U^{\prime}\left(C\left(t_{j}, \tau_{j}\right)\right)=$ $c_{t_{j}^{+}}^{-\alpha}$.

${ }^{15}$ King, Plosser, and Rebelo (1988) have shown that this specification of the utility function is the only specification in a real business cycle model that is consistent with the empirically observed stationarity of hours worked together with nonstationary consumption.

${ }^{16}$ To see that $V(X, S)>\widetilde{V}(X, S)$ in Region I, use the fact that $V(X, S)$ is strictly increasing in $X$ and $S$ to obtain $V^{K}>V^{A}=V^{B}=\widetilde{V}^{B}=\widetilde{V}^{K}$, where $V^{i}$ is the value of $V(X, S)$ at point $i$ and $\widetilde{V}^{j}$ is the value of $\widetilde{V}(X, S)$ at point $j$ in the figure.

${ }^{17}$ If we relax the assumption that $\theta_{X}=\theta_{S}$, then statement $2 \mathrm{c}$ of Proposition 1 implies that the slope of the linear portion of the indifference curve through points $B$ and $A$ is $-\left(1-\psi_{s}\right) \frac{1-\theta_{S}}{1-\theta_{X}}$ while the slope of the dashed line through points $C, B \prime$, and $A$ ' remains - $\left(1-\psi_{s}\right)$. The horizontal intercept of the indifference curve, $\bar{S}$, is $\frac{1}{1-\theta_{S}} \geq 1$ times as large as $\overline{\bar{S}}$, the horizontal intercept of the dashed line through points $C$, $B \prime$, and $A$ ' because starting from $(X, S)=(0, \bar{S})$ the fixed transaction cost moves the allocation $(X, S)$ to $\left(0,\left(1-\theta_{S}\right) \bar{S}\right)=(0, \overline{\bar{S}})$. Therefore, even if $\theta_{X}>\theta_{S}$, so that the linear portion of the indifference curve 
slopes downward more steeply than the dashed line, the linear portion of the indifference curve will not cross the dashed line for any non-negative values of $X$. Also, statement 4c of Proposition 1 implies that the slope of the indifference curve through points $E$ and $F$ is $-\left(1+\psi_{b}\right) \frac{1-\theta_{S}}{1-\theta_{X}}$. The vertical intercept of the indifference curve is $\frac{1}{1-\theta_{X}} \geq 1$ times as large as the vertical intercept of the dashed line through point $D$ and thus the indifference curve does not cross this dashed line for non-negative values of $S$.

${ }^{18}$ In order to obtain the dollar equivalent cost, we use the fact that a utility cost of $\theta_{1} c_{t_{j+1}}^{1-\alpha}$ is equivalent to a reduction of consumption by $\lambda$ where $\left[(1-\lambda)^{1-\alpha}-1\right] \frac{c_{t_{j+1}}^{1-\alpha}}{1-\alpha}=-\theta_{1} c_{t_{j+1}}^{1-\alpha}$. Solving for $\lambda$ gives $\lambda=$ $1-\left[1-(1-\alpha) \theta_{1}\right]^{\frac{1}{1-\alpha}}$. Since $\lambda$ is measured as a fraction of consumption, the observation cost is equal to a fraction $\lambda\left(c_{t_{j+1}} / S_{t_{j}}\right)$ of the value of the investment portfolio. Hence for a consumer who has $10^{6}$ dollars in $S_{t_{j}}$, the observation cost is $\lambda\left(c_{t_{j+1}} / S_{t_{j}}\right) 10^{6}$. This is the number reported in the second column of Table 1.

${ }^{19}$ Eberly (1994) shows that a version of the consumption Euler equation also holds in a model with a fixed cost of adjusting the stock of durables, by considering consumption at consecutive adjustment dates.

${ }^{20}$ It is worth noting that "sufficiently risk-averse" need not require a very high value of $\alpha$. For instance, if the expected equity premium is $\mu-r_{f}=0.04$ and and the standard deviation of the rate of return on equity is $\sigma=0.16$, then any value of $\alpha$ greater than 1.5625 will be sufficiently risk averse.

${ }^{21}$ Recent work by Yuan (2008) has documented that investors appear to react to news that the stock market has reached a new peak.

${ }^{22}$ Let $D$ be the partial derivative of the first term on the right hand side of equation (A.8) with respect to $\tau_{j}$, holding $C\left(t_{j}, \tau_{j}\right)$ fixed. Therefore,

$D=\left[\omega \frac{\theta_{1}(1-\alpha) e^{-\omega \tau_{j}}}{h\left(\tau_{j}\right)}+\left(\frac{\theta_{1}(1-\alpha)}{e^{\omega \tau_{j}}\left[h\left(\tau_{j}\right)\right]^{2}}\right) h^{\prime}\left(\tau_{j}\right)+\alpha \frac{h^{\prime}\left(\tau_{j}\right)}{h\left(\tau_{j}\right)}\left(1-\frac{\theta_{1}(1-\alpha)}{e^{\omega \tau_{j}} h\left(\tau_{j}\right)}\right)\right] \frac{1}{1-\alpha}\left[h\left(\tau_{j}\right)\right]^{\alpha}\left[C\left(t_{j}, \tau_{j}\right)\right]^{1-\alpha}$

Use the facts that $h^{\prime}\left(\tau_{j}\right)=e^{-\omega \tau_{j}}$ and $\omega h\left(\tau_{j}\right)+e^{-\omega \tau_{j}}=1$ to obtain

$$
D=\frac{h^{\prime}\left(\tau_{j}\right)}{h\left(\tau_{j}\right)}\left[\frac{\theta_{1}(1-\alpha)}{h\left(\tau_{j}\right)-\theta_{1}(1-\alpha) e^{-\omega \tau_{j}}}+\alpha\right]\left(1-\frac{\theta_{1}(1-\alpha)}{e^{\omega \tau_{j}} h\left(\tau_{j}\right)}\right) \frac{1}{1-\alpha}\left[h\left(\tau_{j}\right)\right]^{\alpha}\left[C\left(t_{j}, \tau_{j}\right)\right]^{1-\alpha}
$$

Now use the definition of $\Psi\left(\tau_{j}\right)$ in equation (A.17) to obtain

$$
D=\Psi\left(\tau_{j}\right)\left(1-\frac{\theta_{1}(1-\alpha)}{e^{\omega \tau_{j}} h\left(\tau_{j}\right)}\right) \frac{1}{1-\alpha}\left[h\left(\tau_{j}\right)\right]^{\alpha}\left[C\left(t_{j}, \tau_{j}\right)\right]^{1-\alpha} .
$$

${ }^{23}$ If assets could be transferred without any resource costs (i.e., if $\theta_{X}=\theta_{S}=\psi_{s}=\psi_{b}=0$ ), then $m\left(x_{t_{j}}\right)=1$ at all observation dates, and hence can be eliminated from equation (A.43). 


\section{References}

Abel, A. B., J. C. Eberly, and S. Panageas (2007): "Optimal Inattention to the Stock Market," American Economic Review, Papers and Proceedings, 97(2), 244-249.

Baumol, W. J. (1952): "The Transactions Demand for Cash," Quarterly Journal of Economics, $67(4), 545-556$.

Caplin, A. S., And D. F. Spulber (1987): "Menu Costs and the Neutrality of Money," Quarterly Journal of Economics, 102(4), 703-725.

Constantinides, G. M. (1986): "Capital Market Equilibrium with Transaction Costs," Journal of Political Economy, 94(4), 842-862.

Davis, M. H. A., AND A. R. Norman (1990): "Portfolio selection with transaction costs," Mathematics of Operations Research, 15(4), 676-713.

Duffie, D., And T.-S. Sun (1990): "Transactions Costs and Portfolio Choice in a DiscreteContinuous-Time Setting," Journal of Economic Dynamics and Control, 14(1), 35-51.

Eberly, J. C (1994): “Adjustment of Consumers' Durables Stocks: Evidence from Automobile Purchases," Journal of Political Economy, 102(3), 403-436.

Gabaix, X., And D. Laibson (2002): The 6D Bias and the Equity-Premium Puzzle pp. 257-312, NBER macroeconomics annual 2001, Bernanke, Ben S. and Rogoff, Kenneth, eds. MIT Press, Volume 16. Cambridge and London.

Golosov, M., And R. E. Lucas JR. (2007): "Menu Costs and Phillips Curves," Journal of Political Economy, 115(115), 171-199.

Huang, L., And H. Liu (2007): "Rational Inattention and Portfolio Selection," The Journal of Finance, 62(4), 1999-2040.

King, R. G., C. I. Plosser, and S. T. Rebelo (1988): "Production, Growth and Business Cycles: I. The Basic Neoclassical Model," Journal of Monetary Economics, 21(2/3), 195-232.

Merton, R. C. (1971): "Optimum Consumption and Portfolio Rules in a Continuous-Time Model," Journal of Economic Theory, 3(4), 373-413.

Moscarini, G. (2004): "Limited Information Capacity as a Source of Inertia," Journal of Economics Dynamics and Control, 28(10), 2003-2035.

REIs, R. (2006): "Inattentive Consumers," Journal of Monetary Economics, 53(8), 1761-1800. 
Sims, C. A. (2003): "Implications of Rational Inattention," Journal of Monetary Economics, 50(3), 665-690.

Stokey, N. L. (2009): The Economics of Inaction: Stochastic Control Models with Fixed Costs. Princeton University Press, Princeton NJ.

Tobin, J. (1956): "The Interest Elasticity of the Transactions Demand for Cash," Review of Economics and Statistics, 38, 241-247.

Williams, D. (1991): Probability Theory with Martingales. Cambridge Mathematical Textbooks, Cambridge University Press.

Woodford, M. (2008): "Information-Constrained State-Dependent Pricing," Working Paper, Columbia University.

YuAN, Y. (2008): "Attention and Trading," Working paper, University of Iowa - Department of Finance. 